\title{
Two-dimensional NMR Measurement and Point Dipole Model Prediction of Paramagnetic Shift Tensors in Solids
}

\author{
Brennan J. Walder, Krishna K. Dey, Michael C. \\ Davis, Jay H. Baltisberger, and Philip J. Grandinetti
}

A new two-dimensional NMR experiment to separate and correlate the first-order quadrupolar and chemical/paramagnetic shift interactions is described. This experiment, which we call the shifting- $d$ echo experiment, allows a more precise determination of tensor principal components values and their relative orientation. It is designed using the recently introduced symmetry pathway concept. A comparison of the shifting- $d$ experiment with earlier proposed methods is presented and experimentally illustrated in the case of ${ }^{2} \mathrm{H}(I=1)$ paramagnetic shift and quadrupolar tensors of $\mathrm{CuCl}_{2} \cdot 2 \mathrm{D}_{2} \mathrm{O}$. The benefits of the shifting- $d$ echo experiment over other methods are a factor of two improvement in sensitivity and the suppression of major artifacts. From the 2D lineshape analysis of the shifting- $d$ spectrum the ${ }^{2} \mathrm{H}$ quadrupolar coupling parameters are $\left\langle C_{q}\right\rangle=118.1 \mathrm{kHz}$ and $\left\langle\eta_{q}\right\rangle=0.88$, and the ${ }^{2} \mathrm{H}$ paramagnetic shift tensor anisotropy parameters are $\left\langle\zeta_{P}\right\rangle=-152.5 \mathrm{ppm}$ and $\left\langle\eta_{P}\right\rangle=0.91$. The orientation of the quadrupolar coupling principal axis system relative to the paramagnetic shift anisotropy principal axis system is given by $(\alpha, \beta, \gamma)=\left(\frac{\pi}{2}, \frac{\pi}{2}, 0\right)$. Using a simple ligand hopping model the tensor parameters in the absence of exchange are estimated. On the basis of this analysis the instantaneous principal components and orientation of the quadrupolar coupling is found to be in excellent agreement with previous measurements. A new point dipole model for predicting the paramagnetic shift tensor is proposed yielding significantly better agreement than previously used models. In the new model the dipoles are displaced from nuclei at positions associated with high electron density in the singly occupied molecular orbital predicted from ligand field theory. 


\section{CONTENTS}

I. Introduction

II. Experimental $\quad 5$

A. Sample Preparation $\quad 5$

$\begin{array}{ll}\text { B. NMR } & 5\end{array}$

$\begin{array}{ll}\text { C. Data Modeling } & 6\end{array}$

$\begin{array}{ll}\text { III. Theoretical Background } & 6\end{array}$

A. Symmetry Pathways 6

$\begin{array}{ll}\text { B. NMR Interactions } & 9\end{array}$

1. The Magnetic Field at the Nucleus 9

2. Nuclear Shielding and Paramagnetic Shift 11

3. Quadrupolar Interaction 12

IV. Pulse Sequence Methodology 12

A. Hahn- and Solid-Echo Experiments 12

B. Shifting- $p$ echo 13

$\begin{array}{ll}\text { C. Shifting- } d \text { echo } & 17\end{array}$

D. Double-quantum experiment 21

V. Analysis of $\mathrm{CuCl}_{2} \cdot 2 \mathrm{D}_{2} \mathrm{O}$ Spectra 22

A. Hopping model 23

B. Analysis of the paramagnetic shift tensor 26

VI. Summary

A. 2D frequency tenting algorithm for calculating correlated anisotropic lineshapes 36

$\begin{array}{ll}\text { References } & 38\end{array}$ 


\section{INTRODUCTION}

The NMR spectrum of nuclei magnetically coupled to unpaired electrons is characterized by large paramagnetic frequency shifts and enhanced nuclear spin relaxation rates[1-3]. The paramagnetic shifts originate from a short-range through-bond Fermi contact coupling and a longer-range through-space dipolar coupling called the pseudo-contact shift (PCS). The paramagnetic relaxation enhancement (PRE) arises from two contributions: the magnetic field fluctuations arising from thermal fluctuations of the electron spin state and the molecular motion of the paramagnetic site. Both isotropic paramagnetic shifts and PRE have been exploited successfully as long-range distance constraints in solution-state NMR structural studies, particularly in biomolecules[4].

Although Bloembergen's systematic study[5] of the solid-state proton NMR spectrum of $\mathrm{CuSO}_{4} \cdot 5 \mathrm{H}_{2} \mathrm{O}$ dates back to the 1950 s, the applications of solid-state NMR to paramagnetic samples remained relatively unexplored until a number of papers [6-12] appeared around the late 1980s, coinciding with wider availability of robust and relatively high speed commercial magic-angle spinning (MAS) probes. It was discovered that high resolution proton MAS experiments were aided by the larger paramagnetic shifts which turned homonuclear couplings into inhomogeneous broadenings[13, 14]. As MAS spinning speeds increased it became possible to develop solid-state NMR analogs of many solution-state paramagnetic NMR (pNMR) experiments[15]. The use of both PCS and PRE in solid-state NMR increased dramatically over the last decade and has proven powerful in the emerging field of NMR crystallography[16-18].

In one approach[17, 19], the nuclear PCS and PRE are taken as dependent on the distances to and the orientations of a molecular paramagnetic susceptibility tensor, $\boldsymbol{\chi}$, of neighboring unpaired electrons. The general approach in exploiting these effects in structural analyses is to simultaneously determine $\boldsymbol{\chi}$, i.e., its principal components and orientation, starting with best guesses based on literature values. In the solid-state, additional constraints for structural analyses can be found in the paramagnetic shift anisotropy (PSA) tensor on each NMR active nucleus, which arises from the dipolar coupling between the NMR active nucleus and the unpaired electrons. This approach was recently implemented by Pintacuda and coworkers[16] who used the principal components of ${ }^{1} \mathrm{H}$ PSA tensors in polycrystalline 
lanthanide complexes, measured with transferred-echo double resonance (TEDOR), as additional constraints for refining the crystal structure.

Another valuable constraint would be the orientation of the PSA tensor relative to other nuclear spin interaction tensors, such as a heteronuclear dipolar or a quadrupolar coupling. While the relative orientation of tensors can be extracted from analyses of one-dimensional static and MAS patterns[20-22], it is often a problem compounded by low resolution and high degrees of correlated uncertainty among determined parameters. A more precise determination of relative tensor orientations can be achieved with two-dimensional NMR approaches, as first illustrated by Ernst and coworkers[23].

Recently, we developed the COASTER method[24] for separating and correlating the second-order quadrupolar and shift interactions. This approach measures both the principal components of the two tensors as well as their relative orientations. For nuclei experiencing only first-order quadrupolar and paramagnetic shift anisotropies, such as ${ }^{2} \mathrm{H}(I=1)[25-$ 30], the two pulse double-quantum experiment of Vega, Shattuck, and Pines[31] can be employed to separate and correlate the first-order quadrupolar and shift anisotropies. These experiments, however, suffer from severe non-uniform lineshape excitation. To remedy this problem, Antonijevic and Wimperis [32] recently proposed a three-pulse solid-echo sequence for correlating first-order quadrupolar and shift interactions in stationary samples.

The aim of this paper is twofold. First, we describe two-pulse strategies for generating echoes in terms of the recently proposed symmetry pathway formalism[33], a framework that provides a simple picture for understanding many correlation experiments, a framework that provides a simple picture for understanding many correlation experiments. We then proceed to analyze the sequence of Antonijevic and Wimperis[32] in this context, from which we propose a modification that provides a two-fold sensitivity enhancement and eliminates a substantial spectral artifact. We demonstrate the utility of these sequences in obtaining accurate parameters for the ${ }^{2} \mathrm{H}$ paramagnetic shift and quadrupolar coupling tensors for the model compound $\mathrm{CuCl}_{2} \cdot 2 \mathrm{D}_{2} \mathrm{O}$, modulated by the rapid flipping of the heavy water ligands about their $C_{2}$ axes. The results are discussed in the context of this two-fold hopping model which relates the measured NMR parameters to those that would be measured in the absence of deuteron exchange. Second, we closely examine point dipole models for predicting the full paramagnetic shift tensor and propose a modified approach, guided by the shape of the 
singly occupied molecular orbital, that yields excellent agreement with our measured results.

\section{EXPERIMENTAL}

\section{A. Sample Preparation}

A 1 g sample of $\mathrm{CuCl}_{2}\left(97 \%\right.$, Sigma Aldrich) was dehydrated at $175{ }^{\circ} \mathrm{C}$ for two hours and subsequently dissolved into $1 \mathrm{~mL}$ of boiling $\mathrm{D}_{2} \mathrm{O}$ (99.9\% D, Cambridge Isotopes). The dark green syrup was cooled slowly to room temperature and then cooled further near $0{ }^{\circ} \mathrm{C}$. The resulting crystals were washed with cold $\mathrm{D}_{2} \mathrm{O}$ and left to dry in a nitrogen filled glove bag. The crystals were crushed and the resulting sea green powder was stored in a desiccator to prevent further exposure to moisture. The powdered sample was worked into the bottom of a small capillary tube and loaded into the coil without any densification.

\section{B. NMR}

All NMR experiments were performed at room temperature using a single-channel Bruker static-sample wide-line probe on an Avance DMX spectrometer. The nominal field strength was $9.4 \mathrm{~T}$, and the ${ }^{2} \mathrm{H}$ carrier frequency used for all experiments was $61.4960217 \mathrm{MHz}$. A tightly wound solenoid coil $2 \mathrm{~mm}$ in diameter produced an rf field strength of $200 \mathrm{kHz}$ based on the nutation behavior of heavy water $\left(\mathrm{D}_{2} \mathrm{O}\right)$; refining these estimates on the solid led to $\pi / 2$ pulses of $1.24 \mu \mathrm{s}$ and $\pi$ pulses of $2.48 \mu \mathrm{s}$.

The single-quantum pulse sequences used in this work are shown in Figs. 2 and 4 and were performed with identical sampling parameters to facilitate their comparison. A recycle delay of $40 \mathrm{~ms}$ was sufficient for full ${ }^{2} \mathrm{H}$ relaxation. With 2048 transients and $128 t_{1}$ slices, the duration of these experiments was $3 \mathrm{~h}$. A 64-step nested phase cycle was employed for the shifting- $d$ echo experiment, with each pulse phase independently stepped in multiples of $\pi / 2$ and the receiver phase given by $\phi_{R}=\phi_{1}-2 \phi_{2}+2 \phi_{3}$. Phase cycling the shifting- $p$ echo experiment of Antonijevic and Wimperis was handled in the same fashion using $\phi_{R}=-\phi_{1}+0 \phi_{2}+2 \phi_{3}$. The pulse sequence and phase cycling strategy for the doublequantum echo experiment are described in Fig. 6. Due to calculations by Vega and Pines [34] that appreciable excitation of double-quantum coherence occurs even when $\left|\gamma_{I} B_{1}\right|>\left|\omega_{q}\right|$, 
we used the highest pulse powers possible $\left(\nu_{1}=200 \mathrm{kHz}\right)$ for not only the mixing pulses but the excitation pulse as well. In this way, the pulse durations can be kept short, and inflections of the double-quantum to observable coherence transfer profile over the broad pattern are minimized. The optimum duration of the double-quantum excitation pulse at this power level was found to be $4.8 \mu \mathrm{s}$. The conversion and echo shift pulse durations were $1.24 \mu \mathrm{s} .32$ $t_{1}$ points were collected at an effective 36864 scans per slice. The total experiment time was 13 h 25 min.

\section{Data Modeling}

All spectral processing, including affine transformations, were performed with $R M N[35]$. For lineshape analysis, a computer program was written using a stochastic Markov chain accepting new steps based on the Metropolis-Hastings criterion in order to ensure the $\chi^{2}$ minimum was reached[36]. The use of efficient frequency tenting algorithms[37] was an important factor in performing these searches on the multidimensional $\chi^{2}$ surface in a timely manner, especially for the 2D case[38]. For this purpose a new algorithm, described briefly in Appendix A, was developed. Typically, the uncertainties in the best-fit parameters are obtained from the $\chi^{2}$ surface[39]. This approach, however, fails when the spectral model doesn't yield residuals that are Gaussian distributed about zero intensity[40]. Unfortunately, the residuals of the $2 \mathrm{D}$ spectra and especially their 1D projections exhibit this behavior, and

our attempts to refine the spectral model were unsuccessful. The paramagnetic shift tensors from the various dipole source configurations were calculated using Mathematica[41].

\section{THEORETICAL BACKGROUND}

\section{A. Symmetry Pathways}

The Zeeman truncated internal nuclear spin Hamiltonians, here generically labeled as $\lambda$, can each be expanded into components in a spherical basis[2, 33],

$$
H(\Theta)=\sum_{k} \omega_{k} R_{L, 0}^{\{\lambda\}}(\Theta) T_{l, 0}^{\circ}
$$


Here, $\omega_{k}$ is the size of the $k^{\text {th }}$ component contributing to $H(\Theta), R_{L, 0}^{\{\lambda\}}(\Theta)$ are elements of irreducible spherical tensors of rank $L$ in the laboratory frame, describing the spatial dependence of the nuclear spin interaction in the laboratory frame, and $T_{l, 0}^{\circ}$ are irreducible spherical tensor spin operators[42, 43] in the rotating tilted frame[44].

To simplify analyses and discussions we adopt the symmetry pathway approach to describe the details of NMR signal phase evolution[33]. This framework extends the concept of coherence transfer pathways, defining two more fundamental pathways called the spatial pathway and the spin transition pathway to completely describe an NMR experiment. Given a pulse sequence and desired spin system's spatial and spin transition pathways under that sequence, a series of symmetry pathways can be derived which show, at a glance, when and which frequency components for the system will refocus into echoes.

In the symmetry pathway approach, the information in Eq. (1) is recast in terms of transition frequencies, each expressible as sum of components,

$$
\Omega\left(\Theta, m_{i}, m_{j}\right)=\sum_{k} \Omega_{k}\left(\Theta, m_{i}, m_{j}\right)
$$

with each component, $\Omega_{k}\left(\Theta, m_{i}, m_{j}\right)$, as a product of three terms:

$$
\Omega_{k}\left(\Theta, m_{i}, m_{j}\right)=\omega_{k} \cdot \Xi_{L}^{(k)}(\Theta) \cdot \xi_{l}^{(k)}\left(m_{i}, m_{j}\right)
$$

Here, $\omega_{k}$ gives the size of the $k$ th frequency component, and $\Xi_{L}^{(k)}(\Theta)$ and $\xi_{l}^{(k)}\left(m_{i}, m_{j}\right)$ are functions of the sample's spatial orientation, $\Theta$, and the quantized NMR transition, $m_{i} \rightarrow m_{j}$, which we represent henceforth using outer product notation, as in $\left|m_{j}\right\rangle\left\langle m_{i}\right|$. To emphasize different spatial symmetries under $\mathrm{SO}(3)$ we relabel the spatial functions, $\Xi_{L}(\Theta)$, using the upper-case symbols according to:

$$
\begin{gathered}
\mathbb{S}^{\{\lambda\}} \propto R_{0,0}^{\{\lambda\}}, \\
\mathbb{D}^{\{\lambda\}}(\Theta) \propto R_{2,0}^{\{\lambda\}}(\Theta) .
\end{gathered}
$$

While the symmetry pathway approach focuses on the manipulations of the spins in terms of single transitions, we still find it useful to follow the behavior of spin transition functions under the orthogonal rotation subgroup, labeling the spin transition symmetry functions, 
$\xi_{l}\left(m_{i}, m_{j}\right)$, using the lower-case symbols $\mathbb{p}\left(m_{i}, m_{j}\right)$ and $\mathbb{d}\left(m_{i}, m_{j}\right)$ according to:

$$
\begin{aligned}
& \mathbb{P}\left(m_{i}, m_{j}\right)=\left\langle m_{j}\left|T_{1,0}^{\circ}\right| m_{j}\right\rangle-\left\langle m_{i}\left|T_{1,0}^{\circ}\right| m_{i}\right\rangle, \\
& \mathbb{d}\left(m_{i}, m_{j}\right)=\left\langle m_{j}\left|T_{2,0}^{\circ}\right| m_{j}\right\rangle-\left\langle m_{i}\left|T_{2,0}^{\circ}\right| m_{i}\right\rangle,
\end{aligned}
$$

To simplify usage in figures, tables, and discussions of the nuclear spin interactions, we further define

$$
\begin{array}{r}
p_{I}\left(m_{i}, m_{j}\right)=\mathfrak{p}\left(m_{i}, m_{j}\right)=m_{j}-m_{i}, \\
d_{I}\left(m_{i}, m_{j}\right)=\sqrt{\frac{2}{3}} \mathbb{d}\left(m_{i}, m_{j}\right)=m_{j}^{2}-m_{i}^{2} .
\end{array}
$$

Our definition of $p_{I}\left(m_{i}, m_{j}\right)$ is identical to the "coherence order", $p$, defined by Bodenhausen et al.[45], and our definition of $d_{I}\left(m_{i}, m_{j}\right)$ is identical to the "satellite order", $q$, recently defined by Antonijevic and Bodenhausen[46]. The spin transition functions $p_{I}\left(m_{i}, m_{j}\right)$ and $d_{I}\left(m_{i}, m_{j}\right)$ reflect their symmetry under the orthogonal rotation subgroup, which is relevant during rf manipulations in the limit that the rf field strength is larger than all internal frequency contributions. In such a situation, familiar rules hold, such as $d_{I}\left(m_{i}, m_{j}\right)$ values being invariant under a $\pi$ pulse while $p_{I}\left(m_{i}, m_{j}\right)$ change sign. This symmetry is apparent in the transition symmetry tables (see Fig. 1 and Grandinetti et al. [33]) in which inverting the $\left(m_{i}, m_{j}\right)$ values represents the effect of a non-selective $\pi$ pulse.

While the general objective of this work is to develop experiments to separate frequency contributions with spin transition symmetries of $\mathfrak{p}_{I}$ and $\mathbb{d}_{I}$, our specific focus is the separation of the first-order quadrupolar interaction from the nuclear shielding and paramagnetic shift interactions. It is worth noting that homonuclear and heteronuclear dipolar coupling contributions also share similar spin transition symmetries, i.e., $\mathbb{d}_{I I}$ and $\mathbb{d}_{I S}$, respectively, and can be separated from shift ( $\mathbb{p}_{I}$ symmetry) contributions using similar strategies.

While these methods are not appropriate for half-integer nuclei experiencing second-order quadrupolar anisotropies, we have proposed an alternative method called COASTER (Correlation Of Anisotropies Separated Through Echo Refocusing) for separating and correlating the second-order quadrupolar and shift interactions of half-odd integer quadrupolar nuclei in a two-dimensional multiple-quantum variable-angle spinning experiment[24]. 


\section{B. NMR Interactions}

The principal axis system (PAS) of a real second-rank symmetric tensor is defined as the coordinate system where its matrix representation is diagonal with principal components ordered according to the Haeberlen convention[47], $\left|\lambda_{z z}\right| \geq\left|\lambda_{y y}\right| \geq\left|\lambda_{x x}\right|$. The anisotropic lineshape is characterized by the anisotropic strength parameter $\zeta_{\lambda}=\lambda_{z z}$ and asymmetry parameter $\eta_{\lambda}=\left(\lambda_{x x}-\lambda_{y y}\right) / \zeta_{\lambda}$. When multiple anisotropic interactions are present, the resulting anisotropic lineshape will not only depend on the principal components describing each interaction but also the relative orientations of their principal axis systems.

\section{The Magnetic Field at the Nucleus}

Nuclear shielding and paramagnetic shift interactions[48-55] are associated with the spatial variations of the microscopic magnetic field. The magnetostatic description of the sources contributing to the local magnetic field at the nucleus requires a treatment first described by Lorentz[56]. In this treatment the local field at the nucleus is the sum of microscopic magnetic field sources within a spherical "Lorentz cavity" centered at the nucleus as well as from the macroscopic field arising from the continuum magnetization of the bulk crystal outside the cavity. With this picture the local magnetic field at the nucleus can be written[57]

$$
\mathbf{B}_{\text {local }}=\mathbf{B}_{0}-\left[\left(\boldsymbol{N}-\frac{1}{3} \hat{\mathbf{1}}\right) \cdot \boldsymbol{\chi}_{m}\right] \cdot \mathbf{B}_{0}+\mathbf{B}_{\text {cavity }}
$$

where $\mathbf{B}_{0}$ is the externally applied magnetic field, $\boldsymbol{N}$ is the demagnetizing tensor field, $\hat{\mathbf{1}}$ is the unit tensor, $\boldsymbol{\chi}_{m}$ is the bulk magnetic susceptibility, and $\mathbf{B}_{\text {cavity }}$ is microscopic magnetic field generated by sources inside the cavity. Successful application of this treatment requires that the radius of the Lorentz cavity be large enough to render the distinction between discrete and continuous dipole densities at large distances from the center unimportant.

Switching from a single crystal to a polycrystalline sample introduces an additional field, $\mathbf{B}_{\text {dipolar }}$, arising from the dipolar couplings between the bulk magnetization of neighboring crystallites. It can often be assumed that the crystallites are spherical, in which case the demagnetizing field becomes uniform, with $\boldsymbol{N}=\frac{1}{3} \hat{\mathbf{1}}$, and the second term drops out of Eq. (7). With these changes, Eq. (7) reduces to

$$
\mathbf{B}_{\text {local }}=\mathbf{B}_{0}+\mathbf{B}_{\text {dipolar }}+\mathbf{B}_{\text {cavity }} \text {. }
$$



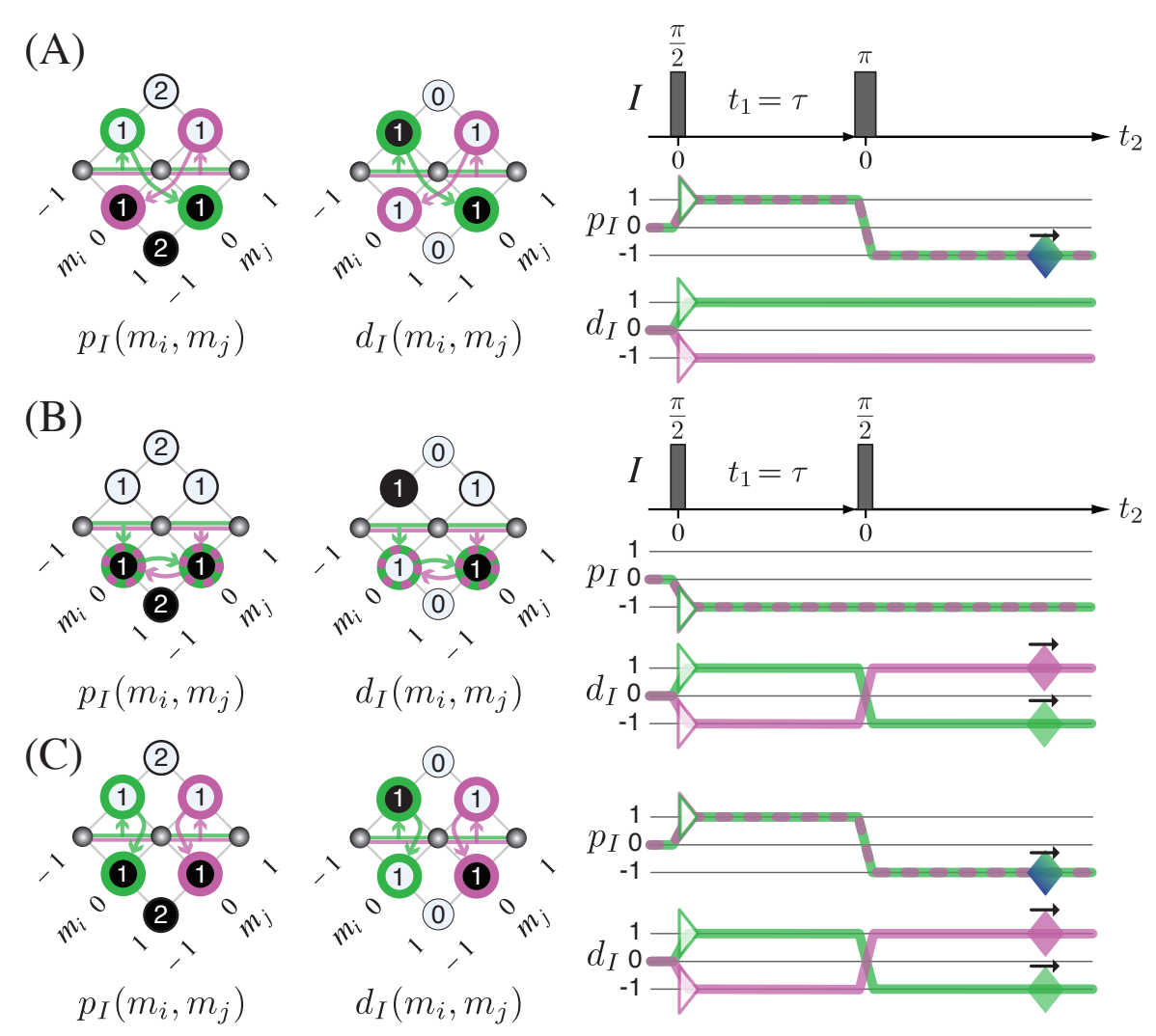

FIG. 1. Spin transition diagrams and pathways for (A) the Hahn-echo, (B) solid-echo, and (C) simultaneous Hahn-echo, solid-echo experiments. Values inside filled black circles are negative. Diamonds indicate pathway refocusing, and are directly observable when filled. Black arrows indicate the direction the echo shifts in time with increasing $t_{1}$.

The contributions to $\mathbf{B}_{\text {dipolar }}$ can be decomposed into those arising from the isotropic part (the IBMS shift) and the anisotropic part (ABMS shift) of $\boldsymbol{\chi}_{m}$ and are primarily functions of crystallite volume, packing, and for the ABMS shift, orientation[58]. For $\mathrm{CuCl}_{2} \cdot 2 \mathrm{H}_{2} \mathrm{O}$, the IBMS and ABMS are characterized by $\left(\chi_{m}\right)_{\text {iso }}=2.66 \times 10^{-4}$ and $\zeta_{\chi_{m}}=-3.49 \times 10^{-5}$, respectively[59], in S.I. units. Previous studies[54, 55] have characterized IBMS and ABMS shifts using a model where spherical crystallites of identical size occupy a cubic lattice with random vacancies. Approximating the local field distribution function as normally distributed and considering lattice occupation probabilities ranging between 0.1 and 0.3 , the spectral isotropic shift is estimated to be near zero with a FWHM broadening of 40 to $60 \mathrm{ppm}$. As we will show, this compares well with the approximately $40 \mathrm{ppm}$ of FWHM broadening in Table II, and is consistent with the preparation and loading of the powder sample. 


\section{Nuclear Shielding and Paramagnetic Shift}

While we can conceptually separate the macroscopic and microscopic contributions to $\mathbf{B}$ via the Lorentz cavity, experimentally separating these contributions is an entirely different matter. Although internal chemical shift references are useful tools in eliminating magnetic susceptibility effects on isotropic shift measurements, no approach exists for eliminating magnetic susceptibility effects on shift anisotropies other than accounting for them with additional line broadening as described above. With these caveats in mind, we describe the nuclear shielding and paramagnetic shift contributions to the NMR transition frequency as arising purely from microscopic contributions of $\mathbf{B}$ within the Lorentz cavity.

The nuclear shielding contribution to the NMR frequency can be written using symmetry pathway notation as the sum of two components

$$
\begin{aligned}
\Omega_{\sigma}^{(1)}\left(\Theta, m_{i}, m_{j}\right)= & -\omega_{0} \sigma_{\text {iso }}\left[\mathbb{S}^{\{\sigma\}} \cdot \mathbb{P}_{I}\left(m_{i}, m_{j}\right)\right] \\
& -\omega_{0} \zeta_{\sigma}\left[\mathbb{D}^{\{\sigma\}}(\Theta) \cdot \mathbb{P}_{I}\left(m_{i}, m_{j}\right)\right],
\end{aligned}
$$

where $\omega_{0}$ is the Larmor frequency, $\sigma_{\text {iso }}$ is the isotropic nuclear shielding and $\zeta_{\sigma}$ is the nuclear shielding anisotropy. Note that both the isotropic and second-rank anisotropic components depend on the same spin transition symmetry function $\mathfrak{p}_{I}\left(m_{i}, m_{j}\right)$.

The paramagnetic shift contribution[18,60,61] to the NMR frequency can be written using symmetry pathway notation as the sum of two components

$$
\begin{aligned}
\Omega_{P}^{(1)}\left(\Theta, m_{i}, m_{j}\right)= & \omega_{0} P_{\text {iso }}\left[\mathbb{S}^{\{P\}} \cdot \mathbb{P}_{I}\left(m_{i}, m_{j}\right)\right] \\
& +\omega_{0} \zeta_{P}\left[\mathbb{D}^{\{P\}}(\Theta) \cdot \mathbb{P}_{I}\left(m_{i}, m_{j}\right)\right],
\end{aligned}
$$

where $P_{\text {iso }}$ is the isotropic paramagnetic shift and $\zeta_{P}$ is the paramagnetic shift anisotropy. Note the leading sign is reversed in Eq. (10) relative to Eq. (9); this establishes that positive paramagnetic shifts correspond to stronger local magnetic fields. The isotropic shift component can contain contributions from both the Fermi contact coupling and the pseudocontact shift. The anisotropic paramagnetic shift arises from the through-space dipolar coupling of the nuclear magnetic moment with the thermally averaged magnetic moments of the unpaired electrons. Since the nuclear shielding and paramagnetic shift contributions have identical products of spatial and spin transition symmetries, the shielding and paramagnetic shift contributions to each spatial-spin product cannot be separated through spin and 
spatial manipulations of the sample alone. Nonetheless, it is sometimes possible to separate the shielding and paramagnetic shift contributions by performing experiments on a sample where the paramagnetic metal sites is replaced with an isostructural diamagnetic metal.

\section{Quadrupolar Interaction}

The first-order quadrupolar coupling contribution to the NMR frequency is purely anisotropic and is written in symmetry pathway notation as

$$
\Omega_{q}^{(1)}\left(\Theta, m_{i}, m_{j}\right)=\omega_{q}\left[\mathbb{D}^{\{q\}}(\Theta) \cdot \mathbb{d}_{I}\left(m_{i}, m_{j}\right)\right]
$$

where $\omega_{q}$ is the quadrupolar splitting, defined as

$$
\omega_{q}=\frac{6 \pi C_{q}}{2 I(2 I-1)} .
$$

Here, $C_{q}$ is the quadrupolar coupling constant, given by $C_{q}=e Q_{\gamma I} \zeta_{q} / 4 \pi \epsilon_{0} h$ in S.I. units, and $\zeta_{q}$ is the electric field gradient (efg) tensor anisotropy. An important feature of the first-order quadrupolar coupling is that it is independent of magnetic susceptibility effects, making the efg tensor an excellent frame of reference for determining the orientation of the paramagnetic shift tensor in a molecular frame.

\section{PULSE SEQUENCE METHODOLOGY}

\section{A. Hahn- and Solid-Echo Experiments}

A natural starting point for our discussion is the Hahn-echo[62], the solid-echo[63, 64], and the Hahn-solid-echo[65] experiments, illustrated in Fig. 1A, 1B, and 1C, respectively. In the context of the symmetry pathway framework, the Hahn-echo is an example of a p-echo while the solid-echo experiment is an example of a $d$-echo.

As mentioned earlier, a $\pi$ rotation causes an inversion of $p_{I}$ and $d_{I}$ values through the $\left(m_{i}, m_{j}\right)$ origin. Thus, a $\pi$ rotation for the second pulse in the Hahn echo experiment leads to a perfect refocusing of $p_{I}$ frequency evolution while $d_{I}$ frequency evolution continues perfectly unaffected by the $\pi$ rotation. As a consequence, with both pulses and receiver behaving 
"ideally" the desired Hahn-echo transition pathway signal can be excited and detected without undesired transition pathway signals present. In other words, no phase cycling should, in principle, be needed to record the Hahn-echo signal. For a nucleus like ${ }^{2} \mathrm{H}$, the Hahnecho signal acquired along the $p_{I^{-}}$echo tops as a function of $t_{1}$ will be modulated by the $d_{I}$ symmetry frequency contributions: the first-order quadrupolar splitting.

In the solid-echo experiment the second pulse is a $\pi / 2$ rotation. This generates two sets of transition pathways, both of which are different from the Hahn-echo transition pathways. The first set, shown in Fig. 1B, leads to the formation of $d_{I}$ echoes with $p_{I}$ frequency evolution unaffected, while the second set, shown in Fig. 1C, leads to the formation of both $p_{I}$ and $d_{I}$ echoes, which in the spin $I=1$ case occur simultaneously. As it is relatively easy to separate signals with different $p_{I}$ pathways using phase cycling techniques [45, 66], the signals arising from these two sets of transition pathways are easily separated. This last statement assumes that both pulses and receiver are behaving "ideally". If that is not the case, then separating the signals from transition pathways in Fig. $1 \mathrm{~A}$ and $1 \mathrm{C}$ can be problematic since they have identical $p_{I}$ pathways. There would be a similar challenge in separating signals from the transition pathways in Fig. 1B from another set of transition pathways not shown which have the same $p_{I}$ pathway but are associated with $d_{I}$ symmetry pathways of $d_{I}=0 \rightarrow-1 \rightarrow-1$ and $d_{I}=0 \rightarrow+1 \rightarrow+1$. Only by eliminating all imperfections in the second pulse rotation can these sets of signals be truly separated. For a nucleus like ${ }^{2} \mathrm{H}$, the solid-echo sequence in Fig. 1B yields a series $d_{I}$-echo tops as a function of $t_{1}$ that will be modulated by the $p_{I}$ symmetry frequency contributions: those due to shift interactions.

In principle, either the sequence in Fig $1 \mathrm{~A}$ or $1 \mathrm{~B}$ could be used as a $2 \mathrm{D}$ experiment to separate and correlate $p_{I}$ and $d_{I}$ evolution. A problem with these two-pulse sequences[67] on a quadrupolar nucleus, however, is that it is not possible to acquire the signals associated with $p_{I}$ and $d_{I}$ symmetry and anti-symmetry pathways, a necessary requirement for obtaining pure 2D absorption mode lineshapes[33, 68-70].

\section{B. Shifting- $p$ echo}

Antonijevic and Wimperis[32] proposed a shifted-echo solution[70] for obtaining a pure

$2 \mathrm{D}$ absorption mode lineshapes in the solid-echo experiment with an additional $\pi / 2$ pulse as 




FIG. 2. Relevant spin transition diagrams for the shifting- $p$ echo experiment, displaying the observable transition pathways given in Eq. (13). Values inside filled black circles are negative. For clarity, only the green pathway, corresponding to the first observable transition, is shown. The purple pathway, evolving simultaneously under the same pulse sequence, follows a path that is the reflection of the green pathway about the vertical axis of the diagram. (B) Pulse sequence and $p_{I}$ and $d_{I}$ symmetry pathways for the experiment. Signal acquisition begins immediately after the final pulse where $t_{2}=-\tau$.

illustrated in Fig. 2. The observable transition pathways for this experiment are

$$
\{I=1\}:\left\{\begin{array}{l}
{\left[z_{I}\right] \rightarrow|0\rangle\left\langle-\left.1\right|_{\frac{t_{1}}{2}} \rightarrow \mid 1\right\rangle\left\langle\left. 0\right|_{\frac{t_{1}}{2}} \rightarrow \mid 0\right\rangle\left\langle\left. 1\right|_{t_{2}},\right.} \\
{\left[z_{I}\right] \rightarrow|1\rangle\left\langle\left. 0\right|_{\frac{t_{1}}{2}} \rightarrow \mid 0\right\rangle\left\langle-\left.1\right|_{\frac{t_{1}}{2}} \rightarrow \mid-1\right\rangle\left\langle\left. 0\right|_{t_{2}}\right.}
\end{array}\right.
$$

the second of which is visualized on the spin transition diagrams in Fig. 2A. The derived $p_{I}$ and $d_{I}$ symmetry pathways for both transitions pathways are shown in Fig. 2B. For $t_{1}=0$, both $p_{I}$ and $d_{I}$ pathways refocus simultaneously at $t_{2}=0$. As $t_{1}$ increases, the $d_{I}$ pathway continues to refocus at $t_{2}=0$, but the time at which the $p_{I}$ pathway refocuses shifts forward in $t_{2}$. For this reason, we will refer to the Antonijevic and Wimperis experiment as the shifting- $p$ echo experiment. The refocusing of the $d_{I}$ components at $t_{2}=0$ for all values of $t_{1}$ means that only the $p_{I}$ (i.e., shift) components evolve parallel to the $t_{1}$ dimension. Similarly, all components with symmetry $p_{I}$ are removed along the diagonal line $t_{2}=t_{1}$, designating 

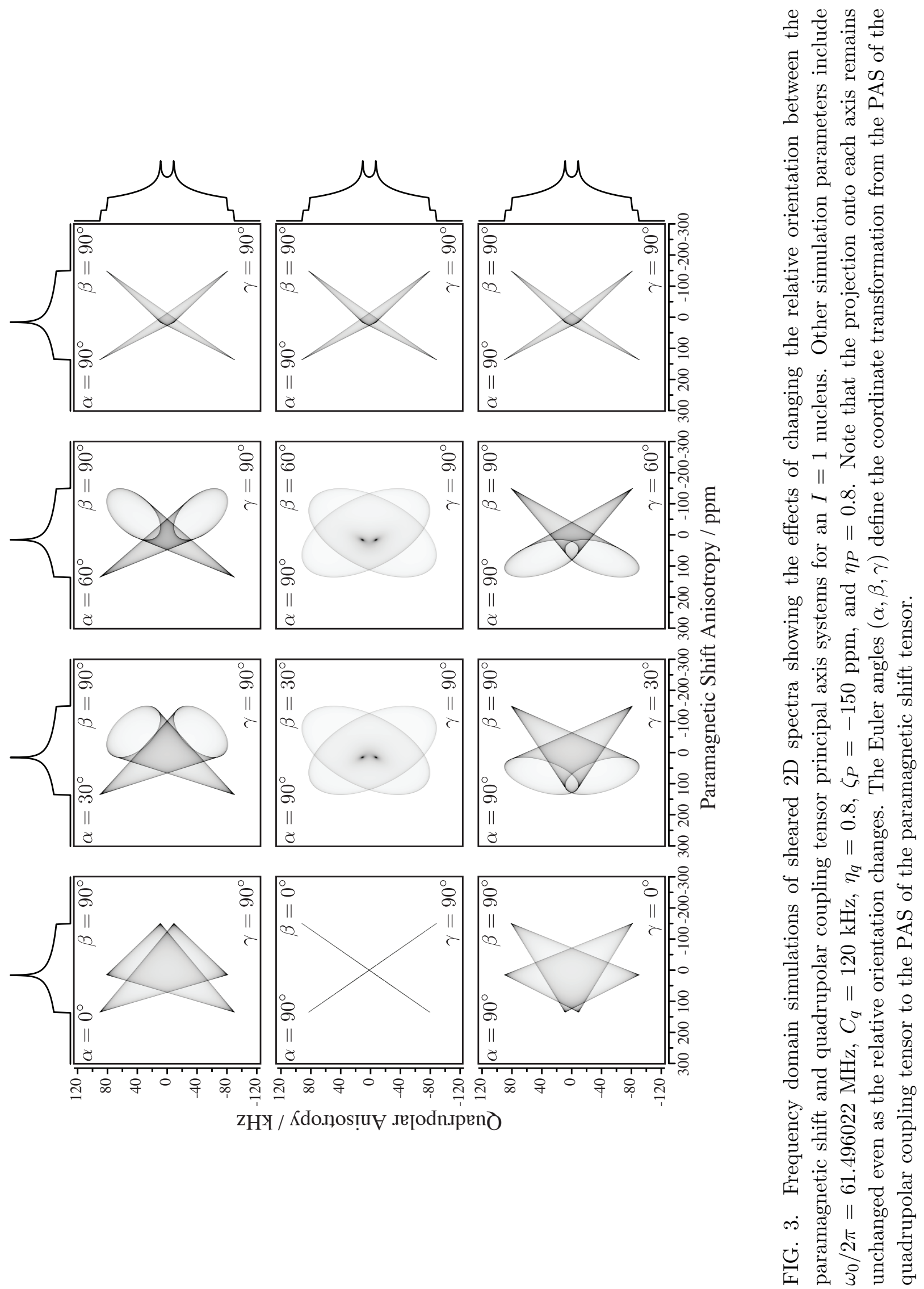
the line parallel to which only pure $d_{I}$ (i.e., first-order quadrupolar) components evolve. Thus, a $45^{\circ}$ skew-projection is needed in the 2D frequency domain signal to obtain a $1 \mathrm{D}$ lineshape arising from pure $d_{I}$ frequency components. The need of a skew projection can be avoided, however, by applying a shear transformation to the $2 \mathrm{D}$ signal parallel to $t_{1}$ with a shear ratio of $\kappa^{\left(t_{1}\right)}=-1$. This leads to a $2 \mathrm{D}$ signal in which the $d_{I}$ components evolve forward along the horizontal $t_{2}^{\prime}$ axis. The shear transformation is then followed by a scaling of $t_{1}$ with $\varsigma^{\left(t_{1}\right)}=-1$ so that $p_{I}$ components appear as $p_{I}=-1$ coherences evolving forward in time along the vertical axis. The 2D FT of the signal after this affine transformation yields a spectrum where the frequency components with $p_{I}$ and $d_{I}$ symmetry are separated into orthogonal dimensions, simplifying spectral interpretation. An advantage of this 2D singlequantum correlation experiment is that 1D projections along the horizontal and vertical coordinates yield two 1D spectra influenced by only one of the two anisotropic interactions. Together, the two 1D projections only lack information about the relative orientations of the two tensors, which can then be determined from the full $2 \mathrm{D}$ correlation spectrum if desired[32, 71].

The 2D spectrum in a spin $I=1$ site consists of two anisotropic 2D lineshapes, each associated with a single transition pathway. These two anisotropic lineshapes are mirror images about zero frequency of the quadrupolar anisotropy dimension. Illustrative examples of $2 \mathrm{D}$ spectra with different tensor orientations are shown in Fig. 3. When the two tensors are diagonal in the same coordinate system the $2 \mathrm{D}$ line shape is a triangular pattern, unless the ratios between corresponding components all have the same value, in which case the pattern in the $2 \mathrm{D}$ spectrum becomes a line segment. The vertices correspond to the principal components of each tensor and establish which components are aligned. When the two tensors are not diagonal in the same coordinate system, an elliptical pattern appears in the $2 \mathrm{D}$ spectrum.

An experimental $2 \mathrm{D}$ spectrum on ${ }^{2} \mathrm{H}$ in a polycrystalline sample of $\mathrm{CuCl}_{2} \cdot 2 \mathrm{D}_{2} \mathrm{O}$ obtained with the shifting- $p$ experiment of Antonijevic and Wimperis is shown in row (I) of Fig. 5A. Discernible in this 2D spectrum is a pair of overlapping triangles, similar to the simulated spectrum in the lower left corner of spectra in Fig. 3, as other studies have analyzed[32, 71]. Notice the intense artifact in the experimental spectrum shown in row (I) of Fig. 5 near the zero frequency in the quadrupolar anisotropy dimension. This artifact[32] arises from tip 
angle errors in the second $\pi / 2$ pulse. In the case of $I=1$, the effect of a $\frac{\pi}{2}+\epsilon$ pulse, which is phase cycled for a $\Delta p_{I}=0$ coherence transfer, on a single-quantum coherence associated with transition $\left|m_{j}\right\rangle\left\langle m_{i}\right|$ is

$$
\begin{aligned}
R_{0}^{-1} & \left(\frac{\pi}{2}+\epsilon\right)\left|m_{j}\right\rangle\left\langle m_{i}\right| R_{0}\left(\frac{\pi}{2}+\epsilon\right) \\
\quad= & \frac{1}{2}\left|-m_{i}\right\rangle\left\langle-m_{j}\right|+\epsilon\left(-\frac{1}{2}\left|m_{j}\right\rangle\left\langle m_{i}\right|\right)
\end{aligned}
$$

to first order in $\epsilon$. Here $R_{\phi}(\beta)$ is the rotation operator characterizing the effect of an rf pulse of tip angle $\beta$ and phase angle $\phi$. We thereby deduce there will be observable and undesired signals from the pathways

$$
\{I=1\}:\left\{\begin{array}{l}
{\left[z_{I}\right] \rightarrow|0\rangle\left\langle-\left.1\right|_{\frac{t_{1}}{2}} \rightarrow \mid 0\right\rangle\left\langle-\left.1\right|_{\frac{t_{1}}{2}} \rightarrow \mid-1\right\rangle\left\langle\left. 0\right|_{t_{2}},\right.} \\
{\left[z_{I}\right] \rightarrow|1\rangle\left\langle\left. 0\right|_{\frac{t_{1}}{2}} \rightarrow \mid 1\right\rangle\left\langle\left. 0\right|_{\frac{t_{1}}{2}} \rightarrow \mid 0\right\rangle\left\langle\left. 1\right|_{t_{2}},\right.}
\end{array}\right.
$$

emerging in proportion to the angle misset $\epsilon$. The $d_{I}$ pathways for this contaminant signal are $d_{I}=0 \rightarrow-1 \rightarrow-1 \rightarrow+1$ and $d_{I}=0 \rightarrow+1 \rightarrow+1 \rightarrow-1$, and lead to the formation of simultaneous and shifting $p_{I}$ and $d_{I}$ echoes that coincide with the desired shifting $p_{I}$ echo pathway in Fig. 2B. In the absence of other interaction symmetries, the simultaneous $p_{I}$ and $d_{I}$ echo completely refocuses all spin evolution and therefore adds a zero frequency component into the quadrupolar anisotropy dimension. Eliminating this artifact here would require that $\left|\gamma_{I} B_{1}\right| \gg \max \left(\left|\omega_{q}\right|,\left|\omega_{0} \zeta_{P}\right|\right)$ and extremely precise pulse calibration, a requirement often difficult to satisfy given breadth of ${ }^{2} \mathrm{H}$ spectra in polycrystalline samples.

\section{Shifting- $d$ echo}

To eliminate the zero-frequency artifact we propose an alternative three pulse experiment by replacing the second $\pi / 2$ pulse with a nonselective $\pi$ pulse to excite the transition pathways

$$
\{I=1\}:\left\{\begin{array}{l}
{\left[z_{I}\right] \rightarrow|-1\rangle\left\langle\left. 0\right|_{\frac{t_{1}}{2}} \rightarrow \mid 1\right\rangle\left\langle\left. 0\right|_{\frac{t_{1}}{2}} \rightarrow \mid 0\right\rangle\left\langle\left. 1\right|_{t_{2}}\right.} \\
{\left[z_{I}\right] \rightarrow|0\rangle\left\langle\left. 1\right|_{\frac{t_{1}}{2}} \rightarrow \mid 0\right\rangle\left\langle-\left.1\right|_{\frac{t_{1}}{2}} \rightarrow \mid-1\right\rangle\left\langle\left. 0\right|_{t_{2}}\right.}
\end{array}\right.
$$

as described in Fig. 4. It is now the $p_{I}$ pathway that always refocuses at $t_{2}=0$ and the $d_{I}$ pathway that refocuses when $t_{2}=t_{1}$. We call this the shifting- $d$ echo experiment. By visualizing this experiment in the same $t_{2}-t_{1}$ coordinate system as before, we see the quadrupolar 


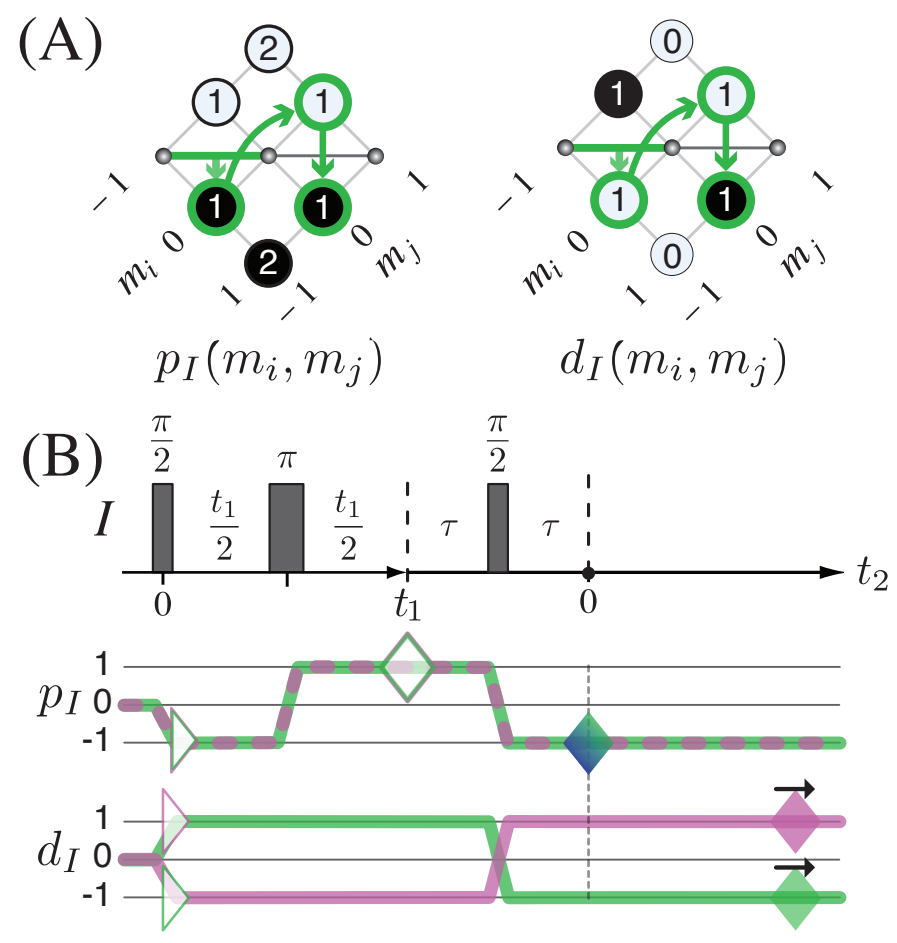

FIG. 4. Relevant spin transition diagrams for the shifting- $d$ echo experiment, displaying the first observable transition pathway given in Eq. (16). Values inside filled black circles are negative. For clarity, only the green pathway, corresponding to the first observable transition, is listed. The purple pathway, evolving simultaneously under the same pulse sequence, follows a path that is the reflection of the green pathway about the vertical axis of the diagram. (B) Pulse sequence and spin transition pathways for the experiment. Signal acquisition begins immediately after the final pulse where $t_{2}=-\tau$.

interaction, of spin symmetry $d_{I}$, now evolving along $t_{1}$; the diagonal line $t_{2}=t_{1}$ now corresponds to pure paramagnetic shift evolution and defines the $t_{2}^{\prime}$ coordinate. The same $\kappa^{\left(t_{1}\right)}=-1$ shearing transformation applied to shifting- $d$ echo signal correlates the two interactions along orthogonal dimensions. A 2D Fourier transform then yields the correlation spectrum.

The experimental 2D spectrum from the shifting- $d$ echo spectrum of $\mathrm{CuCl}_{2} \cdot 2 \mathrm{D}_{2} \mathrm{O}$ appears in row (II) of Fig. 5. The differences between it and the shifting- $p$ echo spectrum extend beyond an apparent transposition of the spectrum. First, the shifting- $d$ experiment does not suffer from a zero-frequency artifact. Again, for $I=1$, the effect of a second $\pi+\epsilon$ pulse now phase cycled for a $\Delta p_{I}=+2$ coherence transfer on a single-quantum coherence associated 




(B)



(II)

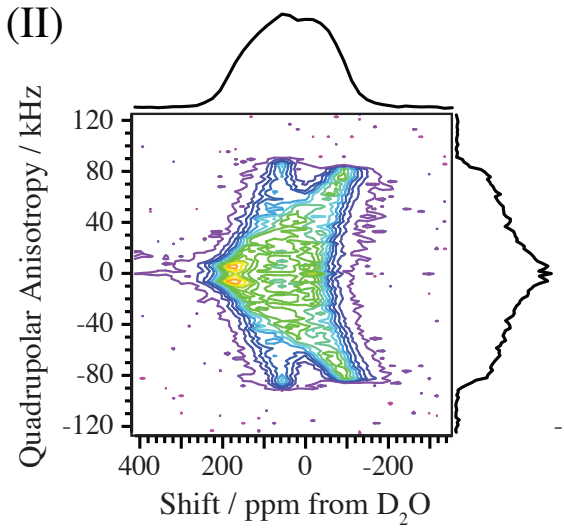

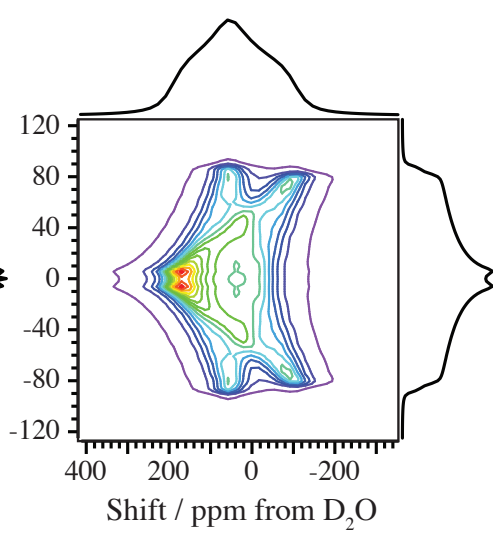

(C)
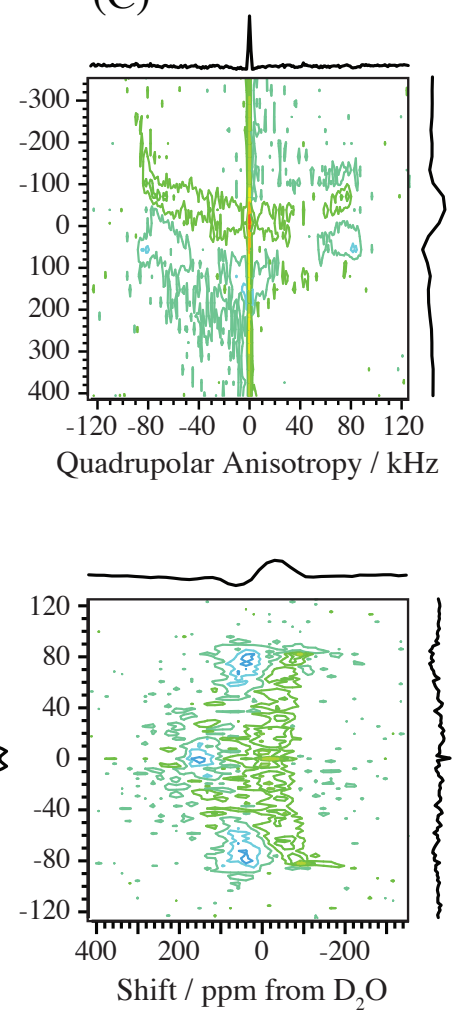

FIG. 5. (A) Experimental spectra, (B) best fit spectra, and (C) best fit residuals for the (I) shifting- $p$ echo and (II) shifting- $d$ echo ${ }^{2} \mathrm{H}$ NMR experiments on polycrystalline $\mathrm{CuCl}_{2} \cdot 2 \mathrm{D}_{2} \mathrm{O}$. The sequences were run with the identical acquisition parameters $\left(\Delta t_{2}=3.4 \mu \mathrm{s}, \Delta t_{1}=4 \mu \mathrm{s}, \tau=135 \mu \mathrm{s}\right)$, and the four plots in (A) and (B) are shown with identical scaling. The response range of the residuals in (C) is also the same as in (A) and (B). Note that the shifting- $d$ experimental spectrum is a factor of two more intense than its counterpart, and does not suffer from the zero quadrupolar frequency artifact plaguing the former.

with transition $\left|m_{j}\right\rangle\left\langle m_{i}\right|$ is

$$
R_{0}^{-1}(\pi+\epsilon)\left|m_{j}\right\rangle\left\langle m_{i}\left|R_{0}(\pi+\epsilon)=\right|-m_{j}\right\rangle\left\langle-m_{i}\right|,
$$

to first order in $\epsilon$. The terms appearing to first order in $\epsilon$ but eliminated by phase cycling for $\Delta p_{I}=+2$ are populations and double quantum coherences. For this reason, the shifting-d echo experiment is more robust in terms of offset effects and pulse length errors, though high rf homogeneity and pulse calibration will still be required for complete artifact elimination.

Second, a $\pi$ rotation inverts the transition, $\left|m_{j}\right\rangle\left\langle m_{i}\right|$ into $\left|-m_{j}\right\rangle\left\langle-m_{i}\right|$ with perfect efficiency, whereas an ideal $\pi / 2$ rotation, in the case of spin $I=1$, transfers the single-quantum coherence associated with the $|m\rangle\langle m \pm 1|$ transition into two transitions of equal amplitude: $|m \pm 1\rangle\langle m|$ and $|-m \mp 1\rangle\langle-m|$. This pathway bifurcation at each $\pi / 2$ pulse means that 



FIG. 6. (A) Spin transition diagrams for the $I=1$ shifted double-quantum echo experiment, displaying the observable transition pathways given in Eq. (18). For clarity, the anti-pathways have been omitted. (B) Pulse sequence and spin transition pathways for the experiment. Signal acquisition begins immediately after the final pulse where $t_{2}=-\tau$. In lieu of hypercomplex acquisition in the manner of States[69], $\phi_{1}, \phi_{2}$, and the receiver phase are cycled for the desired coherence changes, while $\phi$ is incremented in steps of $2 \pi / 8$ as an extra dimensional parameter. FT of this phase dimension allows for easy extraction of the signal along $\Delta p=3$ and $\Delta p=-1$. (C) Shifting double-quantum echo ${ }^{2} \mathrm{H}$ NMR spectrum of polycrystalline $\mathrm{CuCl}_{2} \cdot 2 \mathrm{D}_{2} \mathrm{O}$ composed from path and antipath signal, exhibiting a strong antiphase profile along the quadrupolar dimension $\left(\Delta t_{2}=2 \mu \mathrm{s}\right.$, $\left.\Delta t_{1}=6 \mu \mathrm{s}, \tau=250 \mu \mathrm{s}\right)$.

there are eight transition pathways generated in the shifting- $p$ echo experiment. Only the two pathways of Eq. (13) lead to the desired signal; the remaining six are discarded by phase cycling. By the same token, the shifting-d echo experiment divides the signal into only four pathways, collecting the two listed in Eq. (16). As a result, a signal enhancement factor of two is obtained over the shifting- $p$ echo experiment, as observed by comparing the experimental spectra in Fig. 5A.

A minor disadvantage the shifting- $d$ echo experiment has in comparison is that the excitation profile is expected to be slightly poorer because of substitution of the second $\pi / 2$ pulse by the longer $\pi$ pulse. Nonetheless, in light of the sensitivity improvement and artifact suppression, we anticipate that the shifting- $d$ echo experiment will be preferred over the shifting- $p$ echo experiment. 


\section{Double-quantum experiment}

Any discussion of experiments correlating quadrupolar and shift evolution for $I=1$ nuclei almost demands a comparison to well-established double-quantum techniques. They exploit the symmetry property of $\mathbb{d}_{I}(-m, m)=0$, indicating suppression of the first-order quadrupolar broadening for symmetric $|m\rangle\langle-m|$ transitions. Thus, a period of double-quantum evolution allows one to obtain a spectrum devoid of first-order quadrupolar broadening; however, since $\mathbb{P}_{I}(\mp 1, \pm 1)= \pm 2$, the broadening due to isotropic and anisotropic shift components will be twice as large during double-quantum evolution.

The two-pulse sequence[31] for indirectly detecting double-quantum coherence can, in principle, be used to construct a 2D spectrum that correlates these anisotropies and is capable of producing $2 \mathrm{D}$ absorption mode lineshapes. Doing so requires the $p_{I}=-2 \rightarrow-1$ anti-path be collected in addition to the $p_{I}=+2 \rightarrow-1$ path in order to sample adjacent 2D signal quadrants in $t_{1}[33]$. In practice, however, the resulting spectrum will still be distorted because of the significant loss of signal during receiver dead time. Once again, the simple solution is to generate a shifted-echo signal[70], where the large degree of inhomogeneous broadening can be turned into an advantage through whole-echo acquisition. The best way to achieve this is by appending another non-selective $\pi / 2$ pulse at a time $\tau$ after the mixing pulse to generate the transition pathways:

$$
\begin{gathered}
\left\{\begin{array}{c}
I=1 \\
\text { path }
\end{array}\right\}:\left\{\begin{array}{l}
{\left[z_{I}\right] \rightarrow|1\rangle\left\langle-\left.1\right|_{t_{1}} \rightarrow \mid 0\right\rangle\left\langle-\left.1\right|_{\tau} \rightarrow \mid-1\right\rangle\left\langle\left. 0\right|_{t_{2}},\right.} \\
{\left[z_{I}\right] \rightarrow|1\rangle\left\langle-\left.1\right|_{t_{1}} \rightarrow \mid 1\right\rangle\left\langle\left. 0\right|_{\tau} \rightarrow \mid 0\right\rangle\left\langle\left. 1\right|_{t_{2}},\right.}
\end{array}\right. \\
\left\{\begin{array}{c}
I=1 \\
\text { anti }
\end{array}\right\}:\left\{\begin{array}{l}
{\left[z_{I}\right] \rightarrow|-1\rangle\left\langle\left. 1\right|_{t_{1}} \rightarrow \mid 0\right\rangle\left\langle-\left.1\right|_{\tau} \rightarrow \mid-1\right\rangle\left\langle\left. 0\right|_{t_{2}},\right.} \\
{\left[z_{I}\right] \rightarrow|-1\rangle\left\langle\left. 1\right|_{t_{1}} \rightarrow \mid 1\right\rangle\left\langle\left. 0\right|_{\tau} \rightarrow \mid 0\right\rangle\left\langle\left. 1\right|_{t_{2}},\right.}
\end{array}\right.
\end{gathered}
$$

also illustrated in Fig. 6. Interestingly, few attempts to generate shifted-double-quantum echoes in this manner are found in the literature[72].

By defining the $d_{I}$ echo top, occurring $\tau$ after the final pulse, as the $t_{2}$ origin of a $t_{2}-t_{1}$ coordinate system, clear parallels to the single-quantum correlation experiments discussed previously emerge. The only major difference in comparison to the shifting- $p$ echo experiment evident from the symmetry pathway analysis is that the $t_{2}^{\prime}$ coordinate defining pure quadrupolar evolution advances along the line $t_{2}=2 t_{1}$, so that the affine transformation required to segregate and orient the interactions of differing symmetries is characterized by 
$\kappa^{\left(t_{1}\right)}=-\frac{1}{2}$ and $\varsigma^{\left(t_{1}\right)}=-2$, assuming a pathway signal as defined by Eq. (18). Processing of the anti-pathways defined by Eq. (19) proceeds similarly, utilizing an affine transformation characterized by $\kappa^{\left(t_{1}\right)}=\frac{1}{2}$ and $\varsigma^{\left(t_{1}\right)}=2$.

Similarities in transition symmetry separation strategy do not carry over as similarities between the final single and double-quantum spectra. Since double-quantum excitation is contingent on the tilting of the rotating frame eigenstates by the first-order quadrupolar splitting, the correlation spectrum exhibits a marked dependence on $\omega_{q}$. This is seen strikingly in Fig. 6C, where the node of an antiphase powder pattern is observed at precisely zero frequency in the quadrupolar dimension. Due to calculations by Vega and Pines [34] that appreciable excitation of double-quantum coherence occurs even when $\left|\gamma_{I} B_{1}\right|>\left|\omega_{q}\right|$, we used the highest pulse powers possible $\left(\nu_{1}=200 \mathrm{kHz}\right)$ for not only the mixing pulses but the excitation pulse as well. In this way, the pulse durations can be kept short, and inflections of the double-quantum to observable coherence transfer profile over the broad pattern are minimized, as seen in the experimental spectrum of Fig. 6C. Extracting tensor parameters from these $2 \mathrm{D}$ spectra is not straightforward, since any transfer profile has a value of zero when the first-order quadrupolar splitting goes to zero. Needless to say, when shift anisotropies are significant, the nearly uniform excitation profile offered up by the single quantum correlation experiments make them superior sequences when accurate interaction parameters are desired.

\section{ANALYSIS OF $\mathrm{CuCl}_{2} \cdot 2 \mathrm{D}_{2} \mathrm{O}$ SPECTRA}

The advantage of these 2D correlation experiments is that the principal components of the quadrupolar coupling and paramagnetic shift tensors, as well as their relative orientation, can be obtained with higher precision than earlier approaches. These tensor parameters, described in section III B, can be analyzed in terms of local structure, i.e., bond lengths, bond angles, coordination geometry, crystal symmetry, etc., often with the aid of empirical models and ab initio computational chemistry methods. 


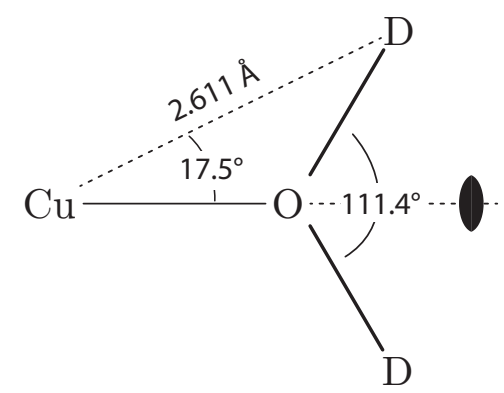

FIG. 7. Orientation of heavy water in paramagnetic $\mathrm{CuCl}_{2} \cdot 2 \mathrm{D}_{2} \mathrm{O}$ crystals. The $\mathrm{D}_{2} \mathrm{O}$ moiety undergoes rapid two-fold hopping about the $\mathrm{Cu}-\mathrm{O}$ bisector, resulting in partial averaging of the tensor components between the two chemically equivalent orientations.

\section{A. Hopping model}

In the case of $\mathrm{CuCl}_{2} \cdot 2 \mathrm{D}_{2} \mathrm{O}$ the analysis is complicated by rapid molecular motion of the water molecule, which occurs on a faster timescale than the NMR experiment, and leads to a spectrum that is characterized by motionally averaged quadrupolar coupling and paramagnetic shift tensors[73, 74]. In the presence of such motional averaging, even if a detailed motional model is known, there will be loss of information about the "instantaneous" tensor, i.e., the tensor in the absence of motion. Thus, the existence and impact of such dynamics must be carefully considered when interpreting measured tensor parameters.

The measured tensor parameters were determined from both the 1D projections and 2D correlation spectrum by chi-squared minimization and are reported in Tables I and II. Because the measured asymmetry parameter is close to unity it is difficult to determine the sign of the paramagnetic anisotropy, $\left\langle\zeta_{P}\right\rangle$. A positive value of $\left\langle\zeta_{P}\right\rangle$ was obtained in the least-squares analysis of the 1D projections, whereas a negative value was found in the least-squares analysis of the 2D spectra. Based on our motional model (vide infra), the Euler angles $\langle\alpha\rangle$ and $\langle\beta\rangle$, describing the relative orientation between the measured quadrupolar and shift tensor principal axis systems, were fixed at $\frac{\pi}{2}$ and the angle $\langle\gamma\rangle$ was found to be within $1^{\circ}$ of zero based on the lineshape analysis.

The water ligands in crystalline hydrates possess internal, rotatory, and translatory vibrational modes[76] which result in slight averaging of the instantaneous NMR parameters $[77,78]$. At room temperature, the ligands also undergo internal deuteron exchange due to the activation of a two-fold hopping motion about the $C_{2}$ site-symmetry axis, as illustrated 


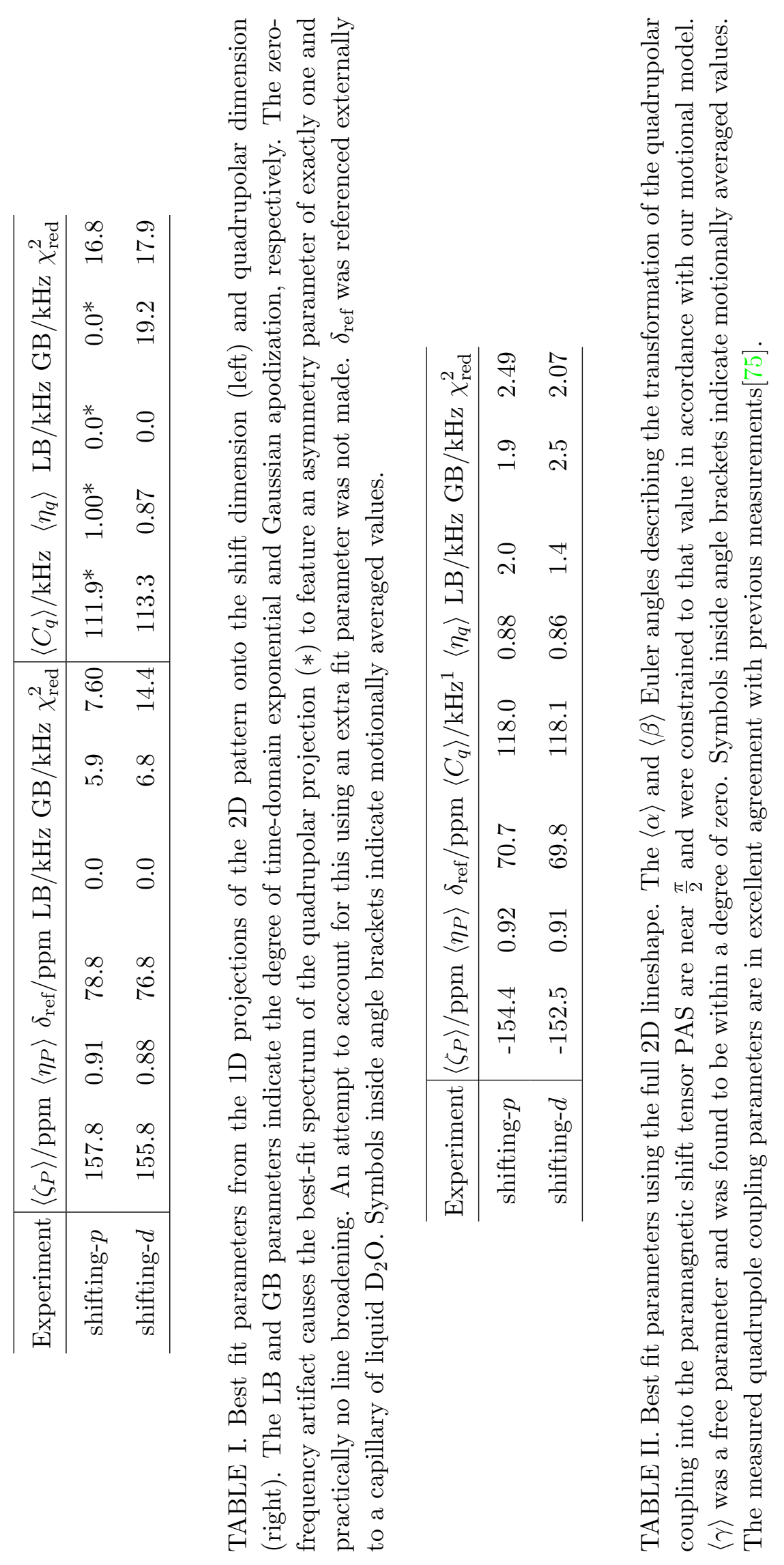


in Fig. 7. Our motional model begins with the assumption that the averaging effects of the vibrations can be ignored. In treating the hopping motion, we will also assume that both orientations are equally probable. We define a molecular frame with its $z$-axis along the $\mathrm{Cu}-\mathrm{O}$ bond and $x z$-plane containing the planar $\mathrm{D}_{2} \mathrm{O}$ molecule. In this frame the average tensor elements are given by

$$
\left\langle R_{2 m}^{\{\lambda\}}\right\rangle=\frac{1}{2}\left[R_{2 m}^{\{\lambda\}}(\Theta)+R_{2 m}^{\prime\{\lambda\}}\left(\Theta^{\prime}\right)\right]
$$

where $\Theta$ and $\Theta^{\prime}$ are the sets of Euler angles transforming the instantaneous tensor PAS of each exchanging site into the molecular frame. In the instantaneous PAS, the spherical components are related to the tensor anisotropy, $\zeta_{\lambda}$, and asymmetry parameters, $\eta_{\lambda}$, according to

$$
\rho_{20}^{\{\lambda\}}=\sqrt{\frac{3}{2}} \zeta_{\lambda}, \quad \rho_{2 \pm 2}^{\{\lambda\}}=\frac{1}{2} \zeta_{\lambda} \eta_{\lambda}
$$

Close examination of either the X-ray[79] or neutron diffraction[80] derived structures of $\mathrm{CuCl}_{2} \cdot 2 \mathrm{D}_{2} \mathrm{O}$ reveals that the two exchanging sites are related by an active rotation about the molecular frame $z$-axis; this leads to the constraints:

$$
\zeta_{\lambda}^{\prime}=\zeta_{\lambda}, \quad \eta_{\lambda}^{\prime}=\eta_{\lambda}
$$

and

$$
\alpha_{\lambda}^{\prime}=\alpha_{\lambda}+\pi, \quad \beta_{\lambda}^{\prime}=-\beta_{\lambda}, \quad \gamma_{\lambda}^{\prime}=\gamma_{\lambda}
$$

Under these constraints, Eq. (20) simplifies to

$$
\begin{aligned}
\left\langle R_{20}^{\{\lambda\}}\right\rangle & =\frac{\zeta_{\lambda}}{2} \sqrt{\frac{3}{2}}\left[3 \cos ^{2} \beta_{\lambda}-1+\eta_{\lambda} \cos 2 \alpha_{\lambda} \sin ^{2} \beta_{\lambda}\right] \\
\left\langle R_{2 \pm 1}^{\{\lambda\}}\right\rangle & =0 \\
\left\langle R_{2 \pm 2}^{\{\lambda\}}\right\rangle & =\frac{\zeta_{\lambda}}{2}\left[\frac{3}{2} \sin ^{2} \beta_{\lambda}+\eta_{\lambda}\left(1-\frac{\sin ^{2} \beta_{\lambda}}{2}\right) \cos 2 \alpha_{\lambda} \mp i \eta_{\lambda} \sin 2 \alpha_{\lambda} \cos \beta_{\lambda}\right] e^{\mp 2 i \gamma_{\lambda}} .
\end{aligned}
$$

The averaged tensor PAS shares an axis with the molecular frame $z$-axis, and thus $\left\langle\alpha_{\lambda}\right\rangle$ and $\left\langle\beta_{\lambda}\right\rangle$ will always be integer multiples of $\pi / 2$. Without additional constraints, Eqs. (24) and (26) cannot be inverted to obtain the five unknown instantaneous tensor parameters. In the case of the deuterium quadrupolar coupling in $\mathrm{D}_{2} \mathrm{O}$, where the $\mathrm{O}-\mathrm{D}$ bond is the dominant contribution to the deuterium efg, we can assume[81] that $\eta_{q}=0$ and that the $z$-axis of the 
instantaneous PAS lies along the O-D bond, i.e., $\alpha_{q}=\gamma_{q}=0$. Then, the nonzero averaged molecular frame Cartesian tensor components become

$$
\begin{aligned}
\left\langle R_{x x}^{\{\lambda\}}\right\rangle & =\frac{\zeta_{\lambda}}{2}\left[3 \sin ^{2} \beta_{\lambda}-1\right], \\
\left\langle R_{y y}^{\{\lambda\}}\right\rangle & =-\frac{\zeta_{\lambda}}{2} \\
\left\langle R_{z z}^{\{\lambda\}}\right\rangle & =\frac{\zeta_{\lambda}}{2}\left[3 \cos ^{2} \beta_{\lambda}-1\right],
\end{aligned}
$$

and the instantaneous quadrupole coupling tensor parameters can be solved from the motionally averaged parameters. Our values for $C_{q}$ and $\beta_{q}$ parameters under this constraint are $219.6 \mathrm{kHz}$ and $56.3^{\circ}$. $\beta_{q}$ compares favorably with half the $\mathrm{H}-\mathrm{O}-\mathrm{H}$ angle of $111.4^{\circ}$ determined by X-ray refined values[79] of neutron scattering measurements[80]. The quadrupole coupling constant determined by nuclear quadrupole resonance on the antiferromagnetic state of $\mathrm{CuCl}_{2} \cdot 2 \mathrm{D}_{2} \mathrm{O}[82]$ is $229.8 \mathrm{kHz}$, in fair agreement with our predicted value for the instantaneous $C_{q}$. The difference between our prediction and the value derived from low temperature measurements is consistent with the former being an extrapolation from a more severely vibrationally averaged measurement. We take this as an indication that neglect of the thermally activated vibrations likely incurs an error of no more than $5 \%$ in the instantaneous parameters.

\section{B. Analysis of the paramagnetic shift tensor}

The anisotropic part of the ${ }^{2} \mathrm{H}$ paramagnetic shift tensor arises from the dipolar couplings between the ${ }^{2} \mathrm{H}$ magnetic dipole moment and the thermally averaged classical magnetic moments, $\left\langle\boldsymbol{\mu}_{e}\right\rangle$, of the fast relaxing electron spins. For the orbitally non-degenerate $\mathrm{Cu}^{2+}$ ion, for which $S=\frac{1}{2}$, the induced moment in the high temperature approximation is given by

$$
\left\langle\boldsymbol{\mu}_{e}\right\rangle=\frac{\mu_{B}^{2}}{4 k_{B} T} \boldsymbol{g} \cdot \boldsymbol{g} \cdot \mathbf{B}_{0},
$$

where $\mu_{B}$ is the Bohr magneton, $k_{B}$ is the Boltzmann constant, $T$ is temperature, and $g$ is the electron $g$-tensor. Because the electron spin density is spatially delocalized, the Hamiltonian can be expressed semiclassically as the coupling of the nuclear magnetic dipole moment, $\boldsymbol{\mu}_{I}$, to a continuous microscopic magnetization $\mathbf{M}_{P}(\mathbf{r})$ due to the unpaired spin 
density (including contributions from all $\mathrm{Cu}$ sites in the Lorentz cavity),

$$
H_{d}=-\frac{\mu_{0}}{4 \pi} \int_{V} \mathbf{M}_{P}(\mathbf{r}) \cdot \boldsymbol{D} \cdot \boldsymbol{\mu}_{I} d \tau
$$

where $\boldsymbol{D}$ is the dipolar coupling tensor, defined in a coordinate system with $\boldsymbol{\mu}_{I}$ at the origin and volume element $d \tau$ at $(x, y, z)$ with $r$ as their separation distance and given by[83]

$$
D_{i k}=\frac{1}{r^{3}}\left(\frac{3 r_{i} r_{k}}{r^{2}}-\delta_{i k}\right) \text {, where }\left(r_{i}, r_{k}=x, y, z\right) \text {. }
$$

In the point dipole approximation, $\mathbf{M}_{P}(\mathbf{r})$ is taken as a discrete sum over pure magnetic dipoles located at $\mathbf{r}_{j}$, according to

$$
\mathbf{M}_{P}(\mathbf{r})=\sum_{j}\left\langle\boldsymbol{\mu}_{e}\right\rangle_{j} \delta^{3}\left(\mathbf{r}_{j}\right)
$$

where $\delta^{3}\left(\mathbf{r}_{j}\right)$ is a three-dimensional Dirac delta function. With this, Eq. (31) reduces to

$$
H_{d}=-\frac{\mu_{0}}{4 \pi} \sum_{j}\left\langle\boldsymbol{\mu}_{e}\right\rangle_{j} \cdot \boldsymbol{D}\left(\mathbf{r}_{j}\right) \cdot \boldsymbol{\mu}_{I},
$$

which is often the starting place for the point dipole approximation of the paramagnetic shift tensor[5]. The averaged magnetic moments of Eq. (30) can be substituted into this expression, transforming it into

$$
H_{d}=-\boldsymbol{\mu}_{I} \cdot \boldsymbol{P} \cdot \mathbf{B}_{0}
$$

where $\boldsymbol{P}$ is the paramagnetic shift tensor, given by

$$
\boldsymbol{P}=\frac{\mu_{0}}{4 \pi} \frac{\mu_{B}^{2}}{4 k_{B} T} \sum_{j} \boldsymbol{g}_{j} \cdot \boldsymbol{g}_{j} \cdot \boldsymbol{D}\left(\mathbf{r}_{j}\right)
$$

and the sum is over all point dipoles inside our Lorentz cavity. Eq. (35) clearly illustrates the parallel between the paramagnetic and chemical shift interactions.

To determine the size of our Lorentz cavity, we performed calculations of the paramagnetic shift tensor using the crystal structure reported by Engberg[79]. Using a $g$-tensor[84] that is diagonal in the molecular frame defined in Fig. 7 with components $g_{x x}=2.102, g_{y y}=2.331$, $g_{z z}=2.047$, transformed into the crystal frame as necessary for the two inequivalent $g$ tensor orientations, we summed over all copper nuclei within a given distance from the ${ }^{2} \mathrm{H}$ nucleus and averaged the result according to Eqs. (24)-(26). The behavior of $\left\langle\zeta_{P}\right\rangle$, shown in Fig. 8, is representative of all tensor components, indicating that a cavity radius greater 
than $20 \AA$, enclosing 298 copper nuclei, should be used to guarantee less than $1 \%$ error from the asymptotic results. Considering all nuclei within $60 \AA$, we obtain for the motionally averaged tensor parameters $\left\langle\zeta_{P}\right\rangle=186.7 \mathrm{ppm}$ and $\left\langle\eta_{P}\right\rangle=0.58$. The positive $\left\langle\zeta_{P}\right\rangle$ conflicts with the negative values determined by our correlation experiments in Table II. While, by convention, $\left\langle\eta_{P}\right\rangle$ cannot exceed unity, a value close to unity is near a sign change in $\left\langle\zeta_{P}\right\rangle$. Though the $\left\langle\eta_{P}\right\rangle$ values in Table II are close to unity, the predicted value of $\left\langle\eta_{P}\right\rangle=0.58$ is not, making the sign discrepancy in $\left\langle\zeta_{P}\right\rangle$ particularly significant.

Following Nayeem and Yesinowski[9] we introduce delocalization of unpaired spin density onto the two chlorine nuclei closest to a given copper center. In this model we define $f_{\mathrm{Cl}}$ as the amplitude fraction of $\left\langle\boldsymbol{\mu}_{e}\right\rangle$ transferred to each chlorine nucleus. Repeating the sum over 298 copper and 596 chlorine nuclei with Nayeem and Yesinowski's value of $f_{\mathrm{Cl}}=7.5 \%$ we still obtain poor agreement with predicted motionally averaged tensor parameters of $\left\langle\zeta_{P}\right\rangle=169.3 \mathrm{ppm}$ and $\left\langle\eta_{P}\right\rangle=0.65$. Like Nayeem and Yesinowski, we also attempted to incorporate the effects of closed-shell nuclear shielding. While this leads to a marginal improvement using one particular estimate of the nuclear shielding tensor[85], we have found the effects using other estimates [86] to be even more insignificant, justifying their continued neglect. Interestingly, Nayeem and Yesinowski[9] performed their sum only over the nine nearest $\mathrm{Cl}-\mathrm{Cu}-\mathrm{Cl}$ segments and obtained $\left\langle\zeta_{P}\right\rangle=170.9 \mathrm{ppm},\left\langle\eta_{P}\right\rangle=0.69$. The fact that their prediction adequately reproduces the intensities of their ${ }^{1} \mathrm{H}$ MAS sideband pattern highlights the challenges not only in modeling the paramagnetic shift tensor but also the difficulties of accurately measuring paramagnetic shift anisotropies from spinning sideband analysis[16].

In light of this poor agreement, we performed a least squares fit for $f_{\mathrm{Cl}}$ that minimizes error in the nontrivial molecular frame spherical components $\left\langle R_{20}^{\{P\}}\right\rangle$ and $\left\langle R_{2-2}^{\{P\}}\right\rangle$ relative to the experimentally derived components. The best fit value of $f_{\mathrm{Cl}}=22.9 \%$ is displayed in Table III, along with the predicted values for the instantaneous and motionally averaged paramagnetic shift tensor parameters. As expected, $\left\langle\eta_{P}\right\rangle$ has been driven toward one, but the agreement remains mediocre, with $12.5 \%$ error in the magnitude of the predicted $\left\langle\zeta_{P}\right\rangle=133.5 \mathrm{ppm}$ and again the wrong sign. Therefore, we conclude that Nayeem and Yesinowski's model with partial delocalization onto the two closest chlorine atoms is inadequate, and introduce additional delocalization, $f_{\mathrm{O}}$, onto the two coordinating oxygen. This model yields significantly better agreement with predicted values of $\left\langle\zeta_{P}\right\rangle=-154.5 \mathrm{ppm}$, and $\left\langle\eta_{P}\right\rangle=0.81$ and 


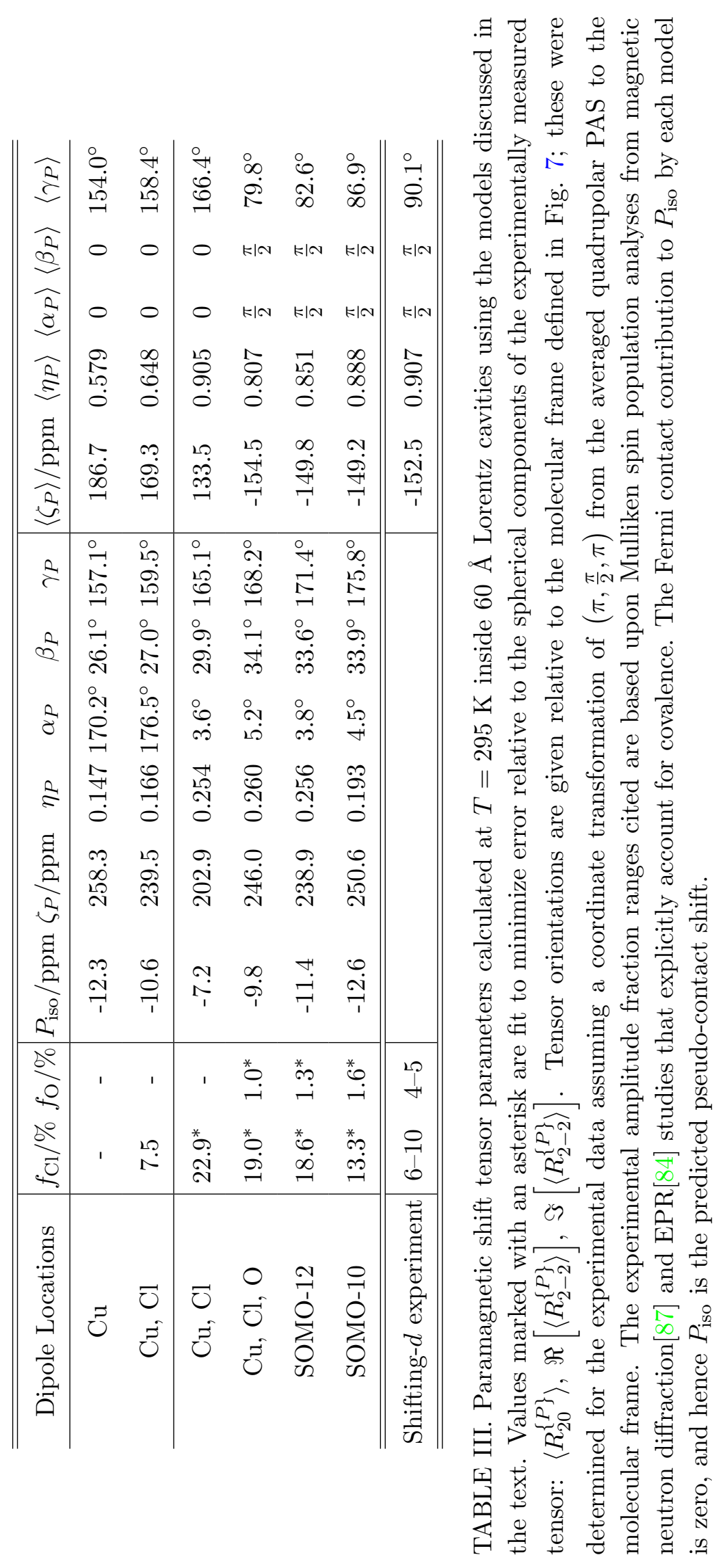






FIG. 8. Behavior of $\left\langle\zeta_{P}\right\rangle$ summed over copper centers as the Lorentz cavity is expanded from $6.25 \AA$ to $60 \AA$. The dashed line indicates the result of a calculation performed in a cavity of 300 $\AA$ radius.

delocalization parameters of $f_{\mathrm{Cl}}=19 \%$ and $f_{\mathrm{O}}=1 \%$. Clearly, the additional delocalization onto oxygen plays a critical role in driving $\left\langle\zeta_{P}\right\rangle$ negative as well as giving physically sensible delocalization parameters. Moreover, they can be compared directly with the point dipole calculations of Rundle[88] using the $\mathrm{CuCl}_{2} \cdot 2 \mathrm{H}_{2} \mathrm{O}$ single-crystal ${ }^{1} \mathrm{H}$ NMR data of Poulis and Hardeman[89] at low temperatures. He concluded that $f_{\mathrm{Cl}}=25 \%$ and $f_{\mathrm{O}}<1 \%$ led to the most consistent interpretation of the available data, in reasonable agreement with our results. That such a small moment distributed to the oxygen has such a substantial influence on these point dipole calculations shows how sensitive the local magnetic field experienced by the deuteron is to the presence of a nearby dipole source, although the discretization of spin density is a much harsher approximation for the water ligand.

Further scrutiny of the tensor parameters reveals that the chlorine-oxygen delocalization model, however, falls short with its predicted $\left\langle\gamma_{P}\right\rangle=79.8^{\circ}$, which deviates from the experimentally determined $\left\langle\gamma_{P}\right\rangle=90.1^{\circ}$. This corresponds to $\left\langle P_{x y}\right\rangle=29.6 \mathrm{ppm}$, showing relatively large residual error remains concealed inside the off-diagonal tensor components, in spite of our model refinements. With this discrepancy in mind, we arrive at our final model where fractional point dipoles are no longer positioned at the nuclei but instead positioned at or near the highest electron densities of each lobe of the singly occupied molecular orbital (SOMO).

We begin this approach by treating the $\mathrm{CuCl}_{2} \cdot 2 \mathrm{D}_{2} \mathrm{O}$ crystal as edge-sharing chains of 
$\left[\mathrm{Cu}\left(\mathrm{D}_{2} \mathrm{O}\right)_{2} \mathrm{Cl}_{4}\right]^{2-}$ clusters individually possessing $D_{2 h}$ point group symmetry. According to ligand field theory, the SOMO, $\psi_{a_{g}^{*}}$, is an anti-bonding orbital of $A_{g}$ symmetry that is predominantly of copper $d_{x^{2}-y^{2}}$ character and interacts with the ligand $p$ orbitals in a sigmalike fashion. The rhombic distortion of the cluster allows us to disregard the contributions of the chloride ligands along the $z$-axis, though it does result in the slight admixture of $d_{z^{2}}$ character, which we can also neglect. In this approximation, all lobes of $\psi_{a_{g}^{*}}$ are directed in the $x y$-plane of the cluster and the probability density can be visualized with a contour plot, as in Fig. 9 where the point dipole positions are shown as black dots. With this model we've increased the number of point dipoles from five to twelve, with four in the lobes of the $d_{x^{2}-y^{2}}$ orbital of $\mathrm{Cu}$ and two in the lobes of each $p$ orbital along the four ligand bonds. From the anti-bonding nature of the SOMO, that is, the destructive interference of atomic wave functions along the bond, we expect two effects on our model. First, the point dipole pair associated with each ligand $p$ orbital becomes asymmetrically delocalized with the point dipole on the bond shifting closer the ligand and taking on a lower fraction of delocalized unpaired electron spin density, whereas the outside point dipole moves further away from the ligand and takes on an increased fraction of the delocalized unpaired electron spin density.

Examination of the local maxima of the atomic wave functions leads to the initial placement of four $3 d_{x^{2}-y^{2}}$ sources $0.4 \AA$ from the copper nucleus, a pair of $3 p$ sources $\pm 0.6 \AA$ along the $x$-axis about each chloride ligand, and a pair of $2 p$ sources placed $\pm 0.2 \AA$ along the $y$-axis about each oxygen nucleus. Next, to model the destructive overlap in the bonding region the chloride sources outside the bond are pushed an additional $0.3 \AA$ away from the center of the cluster, into the channels where water molecules from neighboring chains protrude. We leave the initial assigned sources for oxygen in place, assuming that the tighter $2 p$ orbital reduces the destructive overlap with the copper $3 d$ orbital. A best-fit for $f_{\mathrm{Cl}}$ and $f_{\mathrm{O}}$ using this point dipole placement, shown as SOMO-12 in Table III, has values of $\left\langle\zeta_{P}\right\rangle=-149.8 \mathrm{ppm}$, $\left\langle\eta_{P}\right\rangle=0.85$, and $\left\langle\gamma_{P}\right\rangle=82.6^{\circ}$ with $f_{\mathrm{Cl}}=18.6 \%, f_{\mathrm{O}}=1.3 \%$, yielding better overall agreement with the experimentally measured tensor. Even further improvements can be found by completely eliminating the inner chloride point dipole in the $\mathrm{Cu}-\mathrm{Cl}$ anti-bonding regions, shown as SOMO-10 in Table III, obtaining $f_{\mathrm{Cl}}=13.3 \%, f_{\mathrm{O}}=1.6 \%,\left\langle\zeta_{P}\right\rangle=-149.2 \mathrm{ppm}$, $\left\langle\eta_{P}\right\rangle=0.89$, and $\left\langle\gamma_{P}\right\rangle=86.9^{\circ}$. This yields the lowest overall error in the predicted NMR parameters, which deviate less than $5 \%$ from the measured values. This is similar to the 


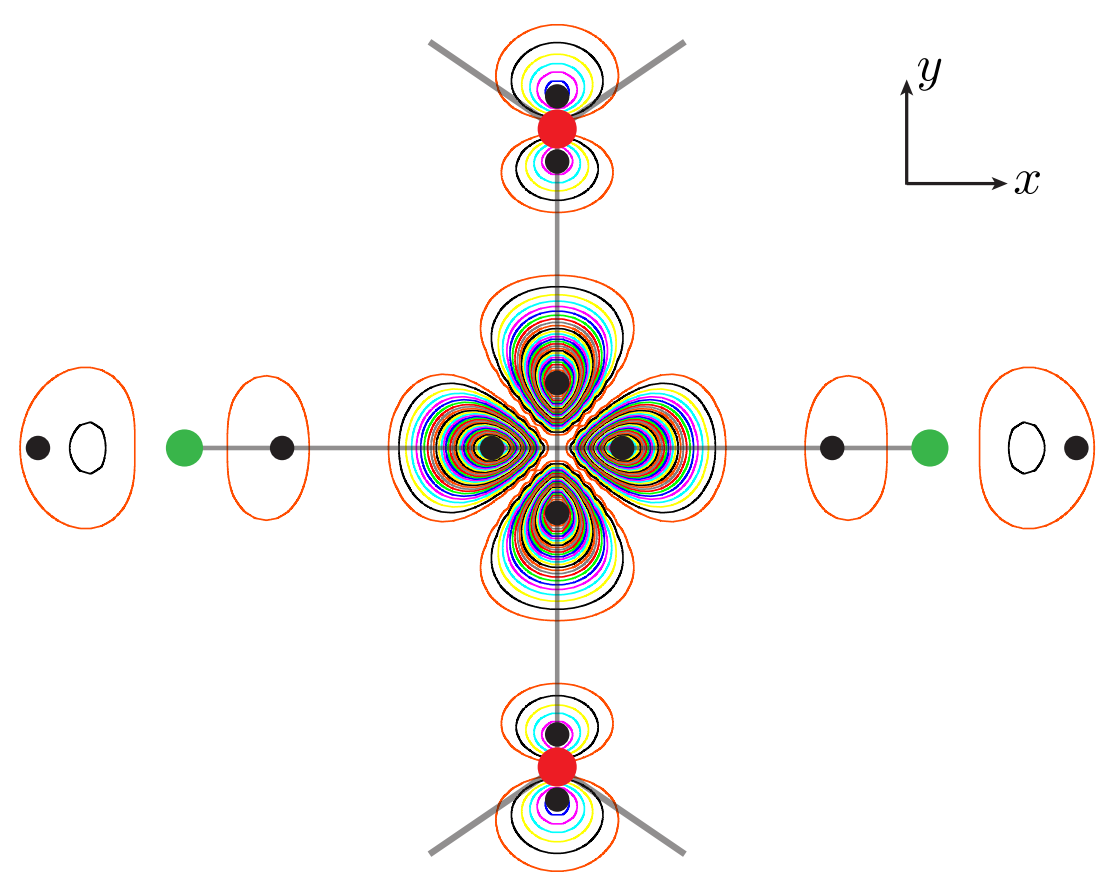

FIG. 9. Schematic for the placement of dipole sources in the SOMO-12 model (black dots). The positions of the chlorine and oxygen nuclei are represented by green and red dots, respectively. The overlaid contours represent the amplitude of the spin density $\rho_{s}=\left|\psi_{a_{g}^{*}}\right|^{2}$ in the $x y$-plane of the $D_{2 h}$ cluster. The atomic orbital coefficients of $\psi_{a_{g}^{*}}$ have been described by Buluggiu et. al[84], and are applied here to single-zeta Slater wave functions[90] for the copper $3 d$, chloride $3 p$, and oxygen $2 p$ orbitals, ignoring contributions due to mixing of the ligand $s$-orbitals.

error we expect from our neglect of water ligand vibrations and approaches the precision limit of our measurements, and thus further refinements of the model are no longer justified.

The fit parameters from the SOMO-10 model are particularly noteworthy. Reynolds and Figgis[87], reanalyzing magnetic neutron diffraction data[91], estimated the spin populations on the chlorine and oxygen atoms to respectively be $6 \%$ and $4 \%$, similar to Mulliken spin populations of $10 \%$ and $5 \%$ that can be deduced from the one-electron wave function characterized by Buluggiu et. al.[84] in their EPR study. Though the ultimate values of $f_{\mathrm{Cl}}$ and $f_{\mathrm{O}}$ we obtain here surely reflect an exaggerated overlap, the fact that they are pulled toward the results of these population analyses lends credibility to the notion that the destructive interference in the bonding regions can be modeled with dipole sources while generating accurate paramagnetic shift tensor parameters.

It is encouraging that our attempt to capture the salient topographical features of SOMO electron density has ultimately driven the predicted paramagnetic shift tensor and the delo- 
calization parameters into better agreement with the available data on this compound. Our findings suggest that with empirical calibration this extension of the MO guided point dipole approximation could prove useful in providing accurate constraints to structure based upon $\zeta_{P}$ and the other anisotropic components of the paramagnetic shift tensor.

This development also invites a truly rigorous computational chemistry approach for calculating paramagnetic shift tensors in solids. Recently, a general formalism has been introduced by Moon and Patchkovskii[92] and expanded upon by Vaara and others[93, 94] for calculating the NMR paramagnetic shift tensor from EPR tensors. Accurately calculating $g$ - and hyperfine coupling tensors from first principles is far from trivial, however, and the development of computational tools for calculating NMR properties from these tensors, particularly for transition metal systems, remains active[95-98]. Moreover, this formalism has been devoted almost exclusively to determining the open-shell contribution to isotropic shifts for molecular systems in solution, being only concerned with the anisotropic components of the $g$ - and hyperfine coupling tensors insofar as they contribute to the contact and pseudo-contact shifts. Yet it is evident in Table III that the pseudo-contact shift behaves quite unlike the other paramagnetic shift tensor parameters, particularly $\zeta_{P}$, in that it is rather insensitive to the exact nature of the microscopic magnetization, and may be a weak proving ground for this class of computational method. To the best of our knowledge, this formalism has yet to be applied to the problem of calculating paramagnetic shift tensor eigenvalues and orientations in solids. This is particularly important for the future development of NMR crystallography in paramagnetic systems[16-18], and it is our belief that our experimental approaches, correlating the paramagnetic shift to other NMR interactions, will play an important role in validating future computational approaches.

\section{SUMMARY}

Using our recently proposed symmetry pathways formalism[33], we have analyzed various two-dimensional NMR methods for separating and correlating the paramagnetic shift and quadrupolar coupling frequency contributions in static polycrystalline samples. Such 2D spectra correlating the ${ }^{2} \mathrm{H}$ paramagnetic shift to the first-order quadrupolar interaction not

only permit the determination of the relative orientation of the two tensors, but the $1 \mathrm{D}$ 
projections along the horizontal and vertical dimensions yield 1D spectra influenced by the separated quadrupolar and paramagnetic shift interactions, respectively. On the basis of our analysis we have proposed a three pulse sequence, called the "shifting- $d$ echo" experiment, that eliminates the spectral distortions of the pre-existing methods while also yielding a two-fold enhancement in sensitivity.

We illustrate our approach on a polycrystalline sample of $\mathrm{CuCl}_{2} \cdot 2 \mathrm{D}_{2} \mathrm{O}$, using a simple motional model of rapid hopping of $\mathrm{D}_{2} \mathrm{O}$ about their $C_{2}$ axes for relating the observed motionally averaged quadrupolar and paramagnetic shift tensors to their instantaneous tensors. For the quadrupolar coupling tensor a simple model for the efg tensor orientation along the $\mathrm{O}-\mathrm{D}$ bond predicts a quadrupolar coupling in excellent agreement with previously measured values. Our analysis of the full deuterium paramagnetic shift tensor reveals that a nuclei-centered point dipole model, which delocalizes the unpaired spin density from the copper onto the two closest chloride, yields poor agreement with our measured values, incorrectly predicting not only the sign of the paramagnetic shift anisotropy but also the tensor orientation. Agreement with the nuclei-centered point dipole model is improved when the unpaired spin density is additionally delocalized onto the two oxygen ligands, yielding the correct sign of the paramagnetic shift anisotropy and closer agreement with the measured tensor orientation. Nearly perfect agreement within the uncertainty of our measurement, however, is found with a model with point dipoles displaced away from the nuclei and at positions consistent with the maximum electron density of the lobes of the singly occupied anti-bonding molecular orbital. Not only does this model yield excellent agreement, but it results in fractional delocalization of $13.3 \%$ onto the two closest chloride and $1.6 \%$ onto the two closest oxygen ligands that is more consistent with electron magnetic resonance[84] and magnetic neutron scattering experiments[87, 91].

Note that the NMR pulse sequences outlined here for static polycrystalline samples will be problematic in multi-site samples due to strong spectral overlap. There have been attempts to apply similar 2D sequences on rotating samples[27] to improve resolution while retaining anisotropy information, but these sequences fail to provide a separation of the shift and quadrupolar frequency contribution onto orthogonal dimensions. Recently, we have proposed a simplified picture[99] for sideband separation experiments and efforts are underway to use the symmetry pathway approach[33] outlined here to extend these MAS approaches to 
separating and correlating shift and quadrupolar anisotropies.

This class of 2D NMR methods, correlating the anisotropic paramagnetic shift interaction to interactions that are independent of magnetic susceptibility effects, such as the first-order quadrupolar coupling, should prove useful in structural studies and NMR crystallography of paramagnetic samples[16-18]. Furthermore, our molecular orbital guided refinements to the point dipole model provide a simple approach for a more accurate prediction of the full paramagnetic shift tensor, i.e., principal component values and tensor orientation. Further work is in progress to determine whether this approach remains valid for complexes with multiple unpaired electrons. 


\section{Appendix A: 2D frequency tenting algorithm for calculating correlated anisotropic lineshapes}

The frequency-domain simulation of a two-dimensional powder pattern correlating anisotropies with an appropriate interpolation scheme can yield an accurate spectrum with fewer crystallite orientations much more quickly than an equivalent time-domain simulation. For one-dimensional anisotropic powder patterns, an effective interpolation scheme is the POWDER method created by Alderman and Grant[37]. In this approach a sphere of $(\theta, \phi)$ angle pairs are constructed as a large number of triangles over the faces of an octahedron inscribed in the unit sphere. The frequency at any given point is determined by interpolating across the triangle vertices via a process called "tenting". In this way, the number of angle pairs required for a smooth powder average is reduced by one or two orders of magnitude.

In the two-dimensional case, the problem is more complicated because vertices of the triangles span two frequency dimensions instead of one. The simplest approach, in which the the vertices are directly binned into the spectrum without interpolation, fails to produce a smooth spectrum output unless a very large number of angle pairs is used. In our algorithm, the Alderman and Grant "tenting" algorithm is modified to allow for an interpolation across both frequency dimensions and bins the signal in the two-dimensional frequency domain spectrum appropriately, as illustrated in Fig. 10. This strategy was previously used by Charpentier and co-workers to develop a frequency-domain simulation of an MQMAS experiment[38]; however, the algorithm they developed suffered from a sub-optimal binning of intensities into the two-dimensional spectrum and produced unsatisfactory patterns. This problem is corrected in the algorithm that we have developed. 

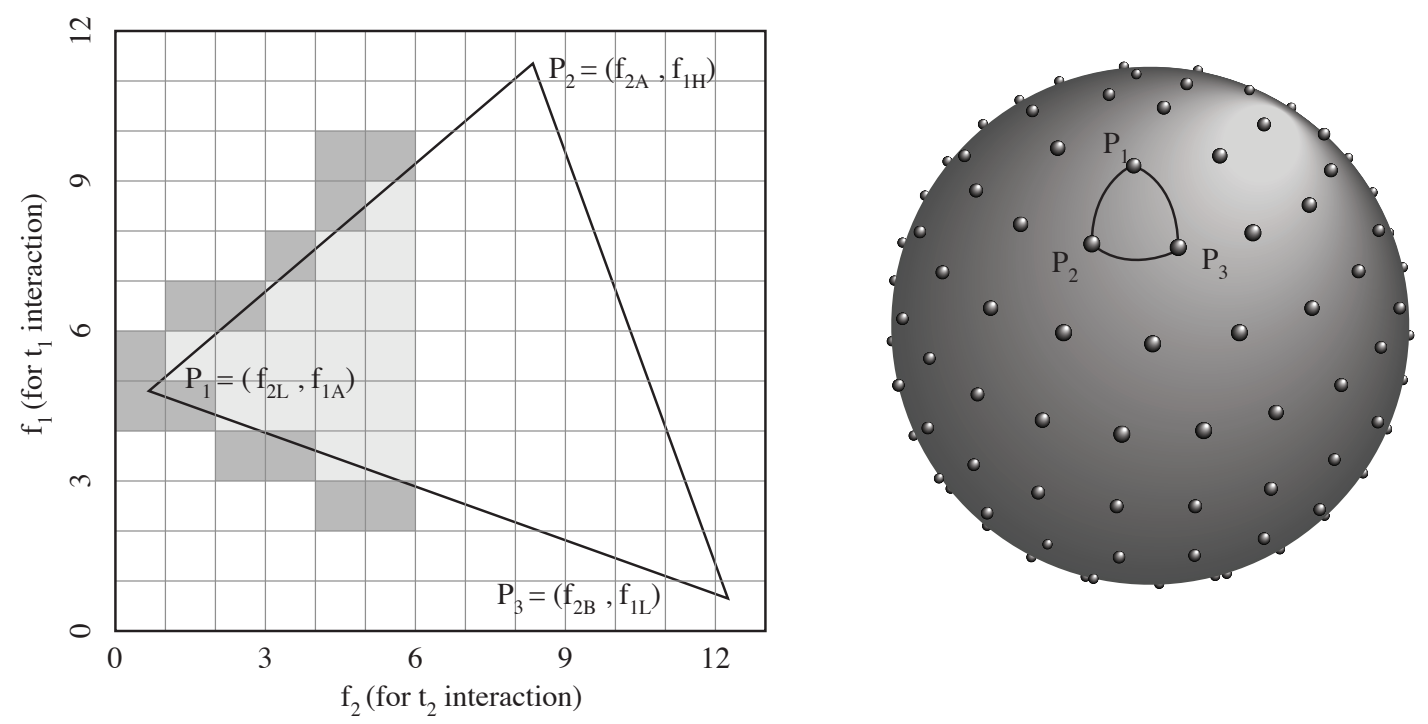

FIG. 10. Contribution of the $(\theta, \phi)$ angle pairs inside the spherical triangle with vertices $\left(P_{1}, P_{2}, P_{3}\right)$ to the two-dimensional correlation spectrum. The spectral intensities are binned into the grid based upon the overlap of each grid square with the triangle formed by interpolating the two-dimensional frequency-domain representation of $\left(P_{1}, P_{2}, P_{3}\right)$. 
[1] C. P. Slichter, Principles of Magnetic Resonance (Springer-Verlag, Berlin, 1980).

[2] A. Abragam, Principles of Nuclear Magnetism (Oxford University Press, Oxford, 1961).

[3] A. Carrington and A. D. McLachlan, Introduction to Magnetic Resonance (Chapman and Hall, Methuen, Inc., 1967).

[4] I. Bertini, C. Luchinat, and G. Parigi, Concepts Magn. Reson. 14, 259 (2002).

[5] N. Bloembergen, Physica 16, 95 (1950).

[6] S. Ganapathy, V. P. Chacko, R. G. Bryant, and M. C. Etter, J. Am. Chem. Soc. 108, 3159 (1986).

[7] J. F. Haw and G. C. Campbell, J. Magn. Reson. 66, 558 (1986).

[8] G. C. Campbell, R. C. Crosby, and J. F. Haw, J. Magn. Reson. 69, 191 (1986).

[9] A. Nayeem and J. P. Yesinowski, J. Chem. Phys. 89, 4600 (1988).

[10] A. K. Cheetham, C. M. Dobson, C. P. Grey, and R. J. B. Jakeman, Nature 328, 706 (1987).

[11] C. P. Grey, C. M. Dobson, A. K. Cheetham, and R. J. B. Jakemant, J. Am. Chem. Soc. 111, 505 (1989).

[12] C. P. Grey, M. E. Smith, A. K. Cheetham, C. M. Dobson, and R. Dupree, J. Am. Chem. Soc. 112, 4670 (1990).

[13] K. Liu, D. Ryan, K. Nakanishi, and A. McDermott, J. Am. Chem. Soc. 117, 6897 (1995).

[14] Y. Ishii, N. P. Wickramasinghe, and S. Chimon, J. Am. Chem. Soc. 125, 3438 (2003).

[15] G. Pintacuda and G. Kervern, Paramagnetic Solid-State Magic-Angle Spinning NMR Spectroscopy, edited by H. Heise and S. Matthews, Topics in Current Chemistry, Vol. 335 (Springer Berlin Heidelberg, Berlin, Heidelberg, 2012) pp. 157 - 200.

[16] G. Kervern, A. D’Aléo, L. Toupet, O. Maury, L. Emsley, and G. Pintacuda, Angew. Chem. Int. Ed. 48, 3082 (2009).

[17] C. Luchinat, G. Parigi, E. Ravera, and M. Rinaldelli, J. Am. Chem. Soc. 134, 5006 (2012).

[18] A. Bhaumik, C. Luchinat, G. Parigi, E. Ravera, and M. Rinaldelli, Cryst. Eng. Comm. 15, 8639 (2013).

[19] I. Bertini, C. Luchinat, G. Parigi, and R. Pierattelli, ChemBioChem 6, 1536 (2005).

[20] T. A. Early, J. Magn. Reson. 74, 337 (1987). 
[21] M. Mizuno, N. Itakura, and K. Endo, Chem. Phys. Lett. 416, 358 (2005).

[22] F. H. Larsen, Solid State Nucl. Mag. 31, 100 (2007).

[23] M. Linder, A. Höhener, and R. R. Ernst, J. Chem. Phys. 73, 4959 (1980).

[24] J. T. Ash, N. T. Trease, and P. J. Grandinetti, J. Am. Chem. Soc. 130, 10858 (2008).

[25] S. E. Woehler, R. J. Wittebort, S. M. Oh, D. N. Hendrickson, D. Inniss, and C. E. Strouse, J. Am. Chem. Soc. 108, 2938 (1986).

[26] T.-H. Lin, J. A. DiNatale, and R. R. Vold, J. Am. Chem. Soc. 116, 2133 (1994).

[27] T. P. Spaniol, A. Kubo, and T. Terao, J. Chem. Phys. 106, 5393 (1997).

[28] T. Iijima, K. Hamada, M. Mizuno, K. Endo, and M. Suhara, J. Phys. Chem. Solids 66, 1101 (2005).

[29] M. Mizuno, Y. Suzuki, K. Endo, M. Murakami, M. Tansho, and T. Shimizu, J. Phys. Chem. A 111, 12954 (2007).

[30] U. G. Nielsen, J. Majzlan, and C. P. Grey, Chem. Mater. 20, 2234 (2008).

[31] S. Vega, T. W. Shattuck, and A. Pines, Phys. Rev. Lett. 37, 43 (1976).

[32] S. Antonijevic and S. Wimperis, J. Chem. Phys. 122, 044312 (2005).

[33] P. J. Grandinetti, J. T. Ash, and N. M. Trease, Prog. Nucl. Mag. Res. Sp. 59, 121 (2011).

[34] S. Vega and A. Pines, J. Chem. Phys. 66, 5624 (1977).

[35] PhySy Ltd, RMN, Version 1.0 (www.physyapps.com, PhySy Ltd, Grandview Heights, OH 43212).

[36] S. Brooks, A. Gelman, G. Jones, and X.-L. Meng, eds., Handbook of Markov Chain Monte Carlo, Chapman \& Hall/CRC Handbooks of Modern Statistical Methods (Chapman and Hall/CRC, Boca Raton, FL, USA, 2011) p. 619.

[37] D. W. Alderman, M. S. Solum, and D. M. Grant, J. Chem. Phys. 84, 3717 (1986).

[38] T. Charpentier, C. Fermon, and J. Virlet, J. Chem. Phys. 109, 3116 (1998).

[39] W. H. Press, S. A. Teukolsky, W. T. Vetterling, and B. P. Flannery, Numerical Recipes (Cambridge University Press, 40 West 20th Street, New York, NY 10011, 1992).

[40] P. J. Brockwell and R. A. Davis, eds., Introduction to Time Series and Forecasting, 2nd ed., Springer Texts in Statistics (Springer New York, New York, NY, 2002) p. 437.

[41] Wolfram Research, Inc., Mathematica, Version 8.0 (Wolfram Research, Inc., Champaign, IL, 2008). 
[42] G. Racah, Phys. Rev. 62, 438 (1942).

[43] H. A. Buckmaster, R. Chatterjee, and Y. H. Shing, Phys. Stat. Sol. A 13, 9 (1972).

[44] N. M. Trease and P. J. Grandinetti, J. Chem. Phys. 128, 052318 (2008).

[45] G. Bodenhausen, H. Kogler, and R. R. Ernst, J. Magn. Reson. 58, 370 (1984).

[46] S. Antonijevic and G. Bodenhausen, J. Magn. Reson. 180, 297 (2006).

[47] R. K. Harris, E. D. Becker, S. M. C. De Menezes, P. Grangerd, R. E. Hoffman, and K. W. Zilm, Solid State Nucl. Mag. 3, 41 (2008).

[48] W. Dickinson, Phys. Rev. Lett. 81, 717 (1951).

[49] L. E. Drain, Proc. Phys. Soc., London 80, 1380 (1962).

[50] D. Vanderhart, W. L. Earl, and A. Garroway, J. Magn. Reson. 44, 361 (1981).

[51] M. Alla and E. Lippmaa, Chem. Phys. Lett. 87, 30 (1982).

[52] A. Garroway, J. Magn. Reson. 49, 168 (1982).

[53] T. M. Barbara, J. Magn. Reson. A 109, 265 (1994).

[54] U. Schwerk, D. Michel, and M. Pruski, J. Magn. Reson., Series A 119, 157 (1996).

[55] A. Kubo, T. P. Spaniol, and T. Terao, J. Magn. Reson. 133, 330 (1998).

[56] H. A. Lorentz, The theory of electrons and its applications to the phenomena of light and radiant heat (Dover Publications, 1915).

[57] J. M. D. Coey, Magnetism and Magnetic Materials (Cambridge University Press, Cambridge, UK, 2010).

[58] One approach to reducing the influence of $\mathbf{B}_{\text {dipolar }}$ is to fill the space between crystallites with a susceptibility matched fluid[100]. This, however, will reintroduce a non-negligible contribution by the demagnetizing field generated by the shape of the sample container[53].

[59] J. van den Handel, H. Gijsman, and N. Poulis, Physica 18, 862 (1952).

[60] H. M. McConnell and R. E. Robertson, J. Chem. Phys. 29, 1361 (1958).

[61] I. Bertini, C. Luchinat, and G. Parigi, Prog. Nucl. Mag. Res. Sp. 40, 249 (2002).

[62] E. L. Hahn, Phys. Rev. 80, 580-594 (1950).

[63] I. Solomon, Phys. Rev. 61, 61 (1958).

[64] J. G. Powles and P. Mansfield, Phys. Lett. 2, 58 (1962).

[65] S. Antonijevic and S. Wimperis, J. Magn. Reson. 164, 343 (2003).

[66] G. P. Drobny, A. Pines, S. Sinton, D. Weitekamp, and D. E. Wemmer, Faraday Symp. Chem. 
Soc. 13, 93 (1978).

[67] J. Davis, K. Jeffrey, M. Bloom, M. Valic, and T. Higgs, Chem. Phys. Lett. 42, 390 (1976).

[68] P. Bachmann, W. Aue, L. Müller, and R. Ernst, J. Magn. Reson. 28, 29 (1977).

[69] D. J. States, R. A. Haberkorn, and D. J. Ruben, J. Magn. Reson. 48, 286 (1982).

[70] P. J. Grandinetti, J. H. Baltisberger, A. Llor, Y. K. Lee, U. Werner, M. A. Eastman, and A. Pines, J. Magn. Reson. A 103, 72 (1993).

[71] S. Antonijevic and S. Persson, J. Chem. Phys. 126, 014504 (2007).

[72] E. Ylinen, A. Kaikkonen, and M. Punkkinen, Solid State Nucl. Mag. 10, 25 (1997).

[73] H. S. Gutowsky and G. E. Pake, J. Chem. Phys. 18, 162 (1950).

[74] K. Larsson, J. Tegenfeldt, and K. Hermansson, J. Chem. Soc., Faraday T. 87, 1193 (1991).

[75] L. C. Gupta and D. L. R. Setty, J. Phys. Soc. Jpn. 26, 1065 (1969).

[76] H. D. Lutz, Bonding and structure of water molecules in solid hydrates. Correlation of spectroscopic and structural data, edited by M. J. Clarke, J. B. Goodenough, J. A. Ibers, C. K. Jørgensen, D. M. P. Mingos, J. B. Neilands, G. A. Palmer, D. Reinen, P. J. Sadler, R. Weiss, and R. J. P. Williams, Structure and Bonding, Vol. 69 (Springer Berlin Heidelberg, Berlin, Heidelberg, 1988) pp. $97-125$.

[77] R. Sjöblom and J. Tegenfeldt, J. Magn. Reson. 27, 405 (1977).

[78] B. Berglund and J. Tegenfeldt, J. Mol. Struct. 40, 139 (1977).

[79] Å. Engberg, Acta Chem. Scand. 24, 3510 (1970).

[80] S. W. Peterson and H. A. Levy, J. Chem. Phys. 26, 220 (1957).

[81] J. Lindgren and J. Tegenfeldt, J. Mol. Struct. 20, 335 (1974).

[82] J. Renard, J. Phys. - Paris 33, 1059 (1972).

[83] Eq. (32) ignores a delta function term present in the classical expression for the dipolar coupling tensor. This term is relevant when the magnetization density overlaps the nucleus, and is the origin of the isotropic Fermi contact coupling.

[84] E. Buluggiu, G. Dascola, D. C. Giori, and A. Vera, J. Chem. Phys. 54, 2191 (1971).

[85] D. Burum and W. Rhim, J. Magn. Reson. 34, 241 (1979).

[86] G. Wu, C. J. Freure, and E. Verdurand, J. Am. Chem. Soc. 120, 13187 (1998).

[87] P. Reynolds and B. Figgis, Phys. Rev. B 42, 2536 (1990).

[88] R. E. Rundle, J. Am. Chem. Soc. 79, 3372 (1957). 
[89] N. Poulis and G. Hardeman, Physica 18, 201 (1952).

[90] J. Slater, Phys. Rev. 36, 57 (1930).

[91] H. Umebayashi, B. Frazer, D. Cox, and G. Shirane, Phys. Rev. 167, 519 (1968).

[92] S. Moon and S. Patchkovskii, "First-principles calculations of paramagnetic NMR shifts," in Calculation of NMR and EPR Parameters: Theory and Applications, edited by M. Kaupp, M. Bühl, and V. G. Malkin (Wiley-VCH, 2004) Chap. 20, pp. 325-338.

[93] T. Pennanen and J. Vaara, Phys. Rev. Lett. 100, 133002 (2008).

[94] W. Van den Heuvel and A. Soncini, J. Chem. Phys. 138, 054113 (2013).

[95] P. Hrobarik, R. Reviakine, A. V. Arbuznikov, O. L. Malkina, V. G. Malkin, F. H. Kohler, and M. Kaupp, J. Chem. Phys. 126, 024107 (2007).

[96] J. Autschbach and B. Pritchard, Theor. Chem. Acc. 129, 453 (2011).

[97] J. Autschbach, S. Patchkovskii, and B. Pritchard, J. Chem. Theory Comput. 7, 2175 (2011).

[98] S. Komorovsky, M. Repisky, K. Ruud, O. L. Malkina, and V. G. Malkin, J. Phys. Chem. A 117, 14209 (2013).

[99] B. J. Walder, K. K. Dey, D. C. Kaseman, J. H. Baltisberger, and P. J. Grandinetti, J. Chem. Phys. 138 (2013), 10.1063/1.4803142.

[100] C. P. Grey, C. M. Dobson, and A. K. Cheetham, J. Magn. Reson. 98, 414 (1992). 